\title{
Spectral changes during dipping in low-mass X-ray binaries due to highly-ionized absorbers
}

\author{
M. Díaz Trigo ${ }^{1}$, A. N. Parmar ${ }^{1}$, L. Boirin ${ }^{2}$, M. Méndez ${ }^{3}$, and J. S. Kaastra ${ }^{3}$ \\ ${ }^{1}$ Research and Scientific Support Department of ESA, ESTEC, Postbus 299, 2200 AG Noordwijk, The Netherlands \\ e-mail:mdiaz@rssd.esa.int \\ 2 Observatoire Astronomique de Strasbourg, 11 rue de l'Université, 67000 Strasbourg, France \\ 3 SRON, National Institute for Space Research, Sorbonnelaan 2, 3584 CA Utrecht, The Netherlands
}

Received 7 June 2005 / Accepted 29 August 2005

\section{ABSTRACT}

X-ray observations have revealed that many microquasars and low-mass X-ray binaries (LMXBs) exhibit narrow absorption features identified with resonant absorption from Fe XXV and Fe XXVI and other abundant ions. In many well studied systems there is evidence for blue-shifts, indicating outflowing plasmas. We succesfully model the changes in both the X-ray continuum and the Fe absorption features during dips from all the bright dipping LMXBs observed by XMM-Newton (EXO 0748-676, XB 1254-690, X 1624-490, MXB 1659-298, 4U 1746-371 and XB 1916-053) as resulting primarily from an increase in column density and a decrease in the ionization state of a highly-ionized absorber in a similar way as was done for XB 1323-619. This implies that the complex spectral changes in the X-ray continua observed from the dip sources as a class can be most simply explained primarily by changes in the highly ionized absorbers present in these systems. There is no need to invoke unusual abundances or partial covering of extended emission regions. Outside of the dips, the absorption line properties do not vary strongly with orbital phase. This implies that the ionized plasma has a cylindrical geometry with a maximum column density close to the plane of the accretion disk. Since dipping sources are simply normal LMXBs viewed from close to the orbital plane this implies that ionized plasmas are a common feature of LMXBs.

Key words. X-rays: individual: EXO 0748-676, XB 1254-690, X 1624-490, MXB 1659-298, 4U 1746-371, XB 1916-053 $\mathrm{X}$-rays binaries - accretion, accretion disks

\section{Introduction}

Around 10 galactic low-mass X-ray binaries (LMXBs) exhibit periodic dips in their X-ray intensity. The dips recur at the orbital period of the system and are believed to be caused by periodic obscuration of a central X-ray source by structure located in the outer regions of a disk resulting from the impact of the accretion flow from the companion star into the disk (White $\&$ Swank 1982). The depth, duration and spectral properties of the dips vary from source to source and from cycle to cycle. The spectral changes during LMXBs dips are complex and cannot be well described by a simple increase in column density of cold absorbing material with normal abundances (e.g., Courvoisier et al. 1986; Smale et al. 1992).

Modeling of these spectral changes provides a powerful means of studying the structure and location of the emitting and absorbing regions in LMXBs. Two approaches have been used. Initially, in the "absorbed plus unabsorbed" approach (e.g., Parmar et al. 1986) the persistent (non-dipping) spectral shape was used to model spectra from dipping intervals. The spectral evolution during dipping was accounted for by a large increase in the column density of the absorbed component, and a decrease of the normalization of the unabsorbed component. The latter decrease was attributed to electron scattering in the absorber. More recently, in the "complex continuum" approach (e.g., Church \& Balucinska-Church 1995), the X-ray emission is assumed to originate from a point-like blackbody, or disk-blackbody component, together with an extended power-law component. This approach primarily models the spectral changes during dipping intervals by the partial and progressive covering of the power-law emission from an extended source. The absorption of the point-like component is allowed to vary independently from that of the extended component, and usually no partial covering is included.

The improved sensitivity and spectral resolution of Chandra and XMM-Newton is allowing narrow absorption features from highly ionized Fe and other metals to be observed from a growing number of X-ray binaries. These features were first detected from the micro-quasars GRO J1655-40 (Ueda et al. 1998; Yamaoka et al. 2001) and GRS 1915+105 (Kotani et al. 2000; Lee et al. 2002). More recent Chandra High-Energy Transmission Grating Spectrometer (HETGS) observations of the black hole candidate H 1743-322 (Miller et al. 2004b) revealed the presence of blue-shifted Fe XXV and Fe XXVI 
absorption features indicative of a highly-ionized outflow. The LMXB systems that exhibit narrow X-ray absorption features are all known dipping sources (see Table 5 of Boirin et al. 2004) except for GX 13+1. This source shows deep blue-shifted Fe absorption features in its HETGS spectrum, again indicative of outflowing material (Ueda et al. 2004).

Boirin et al. (2005) examined the changes in the equivalent widths, EWs, of the Fe XXV and Fe XXVI absorption features seen from XB 1323-619 during persistent and dipping intervals. They found evidence for the presence of lessionized material in the line of sight during dips consistent with a decrease in the photo-ionization parameter, $\log (\xi)$, from $3.9 \pm 0.1 \mathrm{erg} \mathrm{cm} \mathrm{s}^{-1}$ to $3.13 \pm 0.07 \mathrm{erg} \mathrm{cm} \mathrm{s}^{-1}$, while the equivalent hydrogen column density, $N_{\mathrm{H}}^{\mathrm{xabs}}$, of the ionized absorber increased from $(3.8 \pm 0.4) \times 10^{22}$ atom $\mathrm{cm}^{-2}$ to $(37 \pm 2) \times 10^{22}$ atom $\mathrm{cm}^{-2}$. There was also a smaller increase in the equivalent hydrogen column of neutral material, $N_{\mathrm{H}}$, from $(3.50 \pm 0.02) \times 10^{22}$ atom $\mathrm{cm}^{-2}$ to $(4.2 \pm 0.2) \times 10^{22}$ atom $\mathrm{cm}^{-2}$. During persistent intervals almost all the abundant elements, except for Fe, are fully ionized, whilst during dips this is not the case. This results in the presence of absorption edges and many additional absorption features that blend together causing an apparent change in the $1-10 \mathrm{keV}$ continuum measured by XMM-Newton. Boirin et al. (2005) went on to demonstrate that the changes in the source $1-10 \mathrm{keV}$ continuum during dips are consistent with being due primarily to changes in the ionized absorber and that there is no need to invoke unusual abundances or complex continuum models to explain them.

Similar changes in the Fe XXVI and Fe XXV absorption line EWs during dips have been reported from X 1624-490 (Parmar et al. 2002) and XB 1916-053 (Boirin et al. 2004). In the case of MXB 1659-298, whilst Sidoli et al. (2001) report that there is no obvious orbital dependence of the $E W \mathrm{~s}$ of the Fe absorption features, their Fig. 4 shows that the ratio of FeXXV/FeXXVI EWs is at a maximum during dips, consistent with the presence of less-ionized material. As pointed out by Boirin et al. (2005), since highly-ionized absorption features are seen from many other dip sources, changes in ionized absorbers may also explain the overall variations in X-ray spectra observed during LMXB dipping intervals as a class. Here, we examine this prediction by generalizing the analysis performed on XB 1323-619 to all the other bright dipping sources observed by XMM-Newton: EXO 0748-676, XB 1254-690, X 1624-490, MXB 1659-298, 4U 1746-371, and XB 1916-053. The overall properties of these sources are given in Table 1, while the previously reported XMM-Newton results are summarized in Table 2. XB 1323-619 is included for completeness and comparison. The previously bright dip source 4U 1755-338 was observed by XMM-Newton (Angelini \& White 2003), but is too faint for a detailed spectral study and is not discussed further.

\section{Data analysis}

The XMM-Newton Observatory (Jansen et al. 2001) includes three $1500 \mathrm{~cm}^{2} \mathrm{X}$-ray telescopes each with an European Photon Imaging Camera (EPIC) at the focus. Two of the EPIC imaging spectrometers use MOS CCDs (Turner et al. 2001) and
Table 1. Overall properties of the LMXBs studied here. $L_{36}$ is the bestfit $0.6-10 \mathrm{keV}$ unabsorbed luminosity in units of $10^{36} \mathrm{erg} \mathrm{s}^{-1}$ for distances $d$, except for XB 1323-619 where the 0.5-10 keV unabsorbed luminosity is from Boirin et al. (2005). The orbital periods, $P_{\mathrm{orb}}$, are from Ritter \& Kolb (2003). D indicates the presence of dips and E, eclipses.

\begin{tabular}{lcccc}
\hline \hline LMXB & $\begin{array}{c}P_{\text {orb }} \\
(\mathrm{h})\end{array}$ & $\begin{array}{c}L_{36} \\
\left(\mathrm{erg} \mathrm{s}^{-1}\right)\end{array}$ & $\begin{array}{c}d \\
(\mathrm{kpc})\end{array}$ & $\begin{array}{c}\text { Dips/ } \\
\text { Eclipses }\end{array}$ \\
\hline XB 1916-053 & 0.8 & 4.4 & 9.3 & $\mathrm{D}$ \\
XB 1323-619 & 2.9 & 5.2 & 10 & $\mathrm{D}$ \\
EXO 0748-676 & 3.8 & 3.4 & 10 & $\mathrm{D}, \mathrm{E}$ \\
XB 1254-690 & 3.9 & 10.4 & 10 & $\mathrm{D}$ \\
4U 1746-371 & 5.8 & 10.1 & 10.7 & $\mathrm{D}$ \\
MXB 1659-298 & 7.1 & 34.4 & 15 & $\mathrm{D}, \mathrm{E}$ \\
X 1624-490 & 21 & 47.5 & 15 & $\mathrm{D}$ \\
\hline
\end{tabular}

one uses pn CCDs (Strüder et al. 2001). Reflection Grating Spectrometers (RGS, Den Herder et al. 2001) are located behind two of the telescopes. Since the pn has an effective area a factor of $\sim 5$ higher at $7 \mathrm{keV}$ and a better energy resolution than the MOS CCDs, we concentrate on the analysis of pn data. Table 3 gives details of the observations and modes used. The pn was operated in either Timing or Small Window mode. We obtained all data products from the XMM-Newton public archive and reduced them using the Science Analysis Software (SAS) version 6.0.0 and calibration files released on 30 June 2004, except for (1) the XB 1254-690 data, which were reduced using SAS version 5.4.1 and (2) the EXO 0748-676 data which were reduced with version 6.1.0. In order to minimize the effect of any background variations on the extracted spectra, we excluded from the analysis intervals where the $>10 \mathrm{keV}$ pn count rate (one CCD) was $>1 \mathrm{~s}^{-1}$, except for XB 1254-690 (see Sect. 3.3).

In pn Timing mode, only one CCD chip (corresponding to a field of view of $13.6 \times 4.4$ ) is used and the data from that chip are collapsed into a one-dimensional row (4.4) to be read out at high speed, the second dimension being replaced by timing information. This allows a time resolution of $30 \mu \mathrm{s}$, and photon pile-up occurs only for count rates $>1500 \mathrm{~s}^{-1}$, much brighter than any of the sources analyzed here. Pile-up occurs when two or more photons hit the same, or adjacent, pixels during a single CCD read-out cycle, and is accounted for as a single event with an energy that is the sum of the energies of the individual photons. Pile-up reduces the observed flux of a source and makes the spectrum artificially harder than it actually is. Only single and double events (patterns 0 to 4 ) were selected to reduce pile-up effects. Source events were extracted from 53" wide columns centered on the source positions. Background events were obtained from columns of the same width, but centered well away from the sources.

In pn Small Window mode, only a $63 \times 63$ pixel region of the central CCD is read out every $5.7 \mathrm{~ms}$. The count rates of the sources observed in Small Window mode are close to, or above, the $100 \mathrm{~s}^{-1}$ level, above which pile-up effects become important (Strüder et al. 2001). Multiple events that are incorrectly assigned as single should not produce additional line features. 
Table 2. Previously reported emission and absorption features near $7 \mathrm{keV}$ from the LMXBs studied here. $E_{\text {gau }}$ and $E W$ are the energy and equivalent width of the best-fit Gaussian profiles. The continuum model on which the features are superposed is indicated. dbb, bb, pl, and $\mathrm{cpl}$ indicate disk-blackbody, blackbody, power-law and cutoff power-law models, respectively.

\begin{tabular}{|c|c|c|c|c|c|c|c|}
\hline \multirow[t]{2}{*}{ LMXB } & \multirow{2}{*}{$\begin{array}{l}\text { Continuum } \\
\text { model }\end{array}$} & & \multicolumn{3}{|c|}{ Gaussian features near $7 \mathrm{keV}$} & & \multirow[t]{2}{*}{ Reference } \\
\hline & & & Absorption & & Emis & sion & \\
\hline & & Ident. & $E_{\text {gau }}(\mathrm{keV})$ & $E W(\mathrm{eV})$ & $E_{\text {gau }}(\mathrm{keV})$ & $E W(\mathrm{eV})$ & \\
\hline XB 1916-053 & $\mathrm{dbb}+\mathrm{pl}$ & $\begin{array}{l}\text { Fe XXV } \\
\text { Fe XXVI }\end{array}$ & $\begin{array}{l}6.65_{-0.02}^{+0.05} \\
6.95_{-0.04}^{+0.05}\end{array}$ & $\begin{array}{l}30_{-8}^{+12} \\
30_{-11}^{+12}\end{array}$ & & & Boirin et al. (2004) \\
\hline XB $1323-619$ & $\mathrm{bb}+\mathrm{pl}$ & $\begin{array}{l}\text { Fe XXV } \\
\text { Fe XXVI }\end{array}$ & $\begin{array}{l}6.68 \pm 0.04 \\
6.97 \pm 0.05\end{array}$ & $\begin{array}{l}25_{-7}^{+19} \\
24_{-7}^{+21}\end{array}$ & $6.6_{-0.2}^{+0.1}$ & & Boirin et al. (2005) \\
\hline XB 1254-690 & $\mathrm{dbb}+\mathrm{pl}$ & $\begin{array}{l}\text { Fe XXVI Ly } \alpha \\
\text { Fe XXVI Ly } \beta\end{array}$ & $\begin{array}{l}6.95 \pm 0.03 \\
8.20_{-0.10}^{+0.05}\end{array}$ & $\begin{array}{l}27_{-11}^{+8} \\
17 \pm 9\end{array}$ & & & Boirin \& Parmar (2003) \\
\hline MXB 1659-298 & $\mathrm{bb}+\mathrm{cpl}$ & $\begin{array}{l}\text { Fe XXV } \\
\text { Fe XXVI }\end{array}$ & $\begin{array}{l}6.64 \pm 0.02 \\
6.90_{-0.01}^{+0.02}\end{array}$ & $\begin{array}{l}33_{-9}^{+20} \\
42_{-8}^{+13}\end{array}$ & $6.47_{-0.14}^{+0.18}$ & $160_{-40}^{+60}$ & Sidoli et al. (2001) \\
\hline X 1624-490 & $\mathrm{bb}+\mathrm{pl}$ & $\begin{array}{l}\text { Fe XXV } \\
\text { Fe XXVI }\end{array}$ & $\begin{array}{l}6.72 \pm 0.03 \\
7.00 \pm 0.02\end{array}$ & $\begin{array}{l}7.5_{-1.7}^{+6.3} \\
16.6_{-1.9}^{+5.9}\end{array}$ & $6.58_{-0.04}^{+0.07}$ & $78_{-6}^{+19}$ & Parmar et al. (2002) \\
\hline
\end{tabular}

Table 3. Observation $\log$ for the EPIC pn camera. $T$ is the net exposure time and $C R$ is the pn $0.6-10 \mathrm{keV}$ persistent emission count rate. SW indicates Small Window mode.

\begin{tabular}{lcclll}
\hline \hline LMXB & $\begin{array}{c}\text { Start time } \\
(\text { yr mon dy h:mn) }\end{array}$ & $\begin{array}{c}T \\
(\mathrm{ks})\end{array}$ & Mode & Filter & $\begin{array}{l}C R \\
\left(\mathrm{~s}^{-1}\right)\end{array}$ \\
\hline XB 1916-053 & 2001 Sep. 25 04:14 & 16.5 & Timing & Medium & 75 \\
EXO 0748-676 & 2003 Nov. 12 08:23 & 94.6 & SW & Medium & 30 \\
XB 1254-690 & 2001 Jan. 22 15:49 & 17.6 & Timing & Thin1 & 190 \\
4U 1746-371 & 2002 Sep. 19 11:59 & 45.6 & SW & Medium & 110 \\
MXB 1659-298 & 2001 Feb. 20 08:28 & 31.6 & SW & Thin1 & 160 \\
X 1624-490 & 2001 Feb. 12 07:29 & 57.5 & SW & Medium & 75 \\
\hline
\end{tabular}

We used the SAS task epatplot, which utilizes the relative ratios of single- and double-pixel events which deviate from standard values in case of significant pile-up, as a diagnostic tool in the pn camera Small Window mode data. As expected, this showed that the persistent spectra extracted for all the sources observed in Small Window mode were affected by pile-up. We investigated further the importance of properly correcting for count pile-up in Small Window mode by extracting events in annuli of inner radii 15,20 , and $25^{\prime \prime}$ and an outer radius of $30^{\prime \prime}$ centered on the PSF (point spread function) core for MXB 1659-298. This source was chosen because it has the highest count rate in Small Window mode (see Table 3). We compared then the results of spectral fits to those obtained when the PSF core was included. We obtained consistent spectral results once events within a radius of $15^{\prime \prime}$ were excluded. We repeated a similar process for all the sources affected by pile-up and excluded events from the inner 9!'25, 17'.5, and $15^{\prime \prime}$ radius core of the point spread function (PSF) from the pn spectra of EXO 0748-676, 4U 1746-371 and MXB 1659-298, respectively. In order not to produce any additional uncertainties, we used the same extraction regions for the dip spectra for these sources.

Figure 1 shows the EPIC pn 0.6-10 keV lightcurves of the sources studied here. All the sources except for 4U 1746-371 show deep dipping activity. Regular eclipses are visible from EXO 0748-676 and MXB 1659-298. XMM-Newton has made a number of observations of EXO 0748-676 and we chose the 2002 November 12 observation since the dips appeared to be unusually prominent. X 1624-490 appears to be already in a deep dip at the start of the observation, and only one dip is seen from XB 1254-690 close to the end of the observation. We first excluded X-ray bursts and eclipses from the lightcurves and then selected intervals flagged with thick horizontal lines in Fig. 1 for the extraction of dip spectra and dipfree intervals for the persistent emission spectra. Within the dipping intervals, we extracted between 1 and 5 spectra for each source based on the intensity selection criteria given in Table 4. EXO 0748-676 and MXB 1659-298 spectra with dip depths $>80 \%$ of the persistent emission were excluded from the analysis since they are strongly influenced by background counts.

\section{Spectral results}

We performed spectral analysis using XSPEC (Arnaud 1996) version 11.2, and SPEX (Kaastra et al. 1996) version 2.00.11. We used throughout the photo-electric cross sections of Morrison \& McCammon (1983) to account for absorption by neutral gas with solar abundances (abs model within SPEX, and wabs model in XSPEC). Spectral uncertainties are given at $90 \%$ confidence $\left(\Delta \chi^{2}=2.71\right.$ for one interesting parameter), 

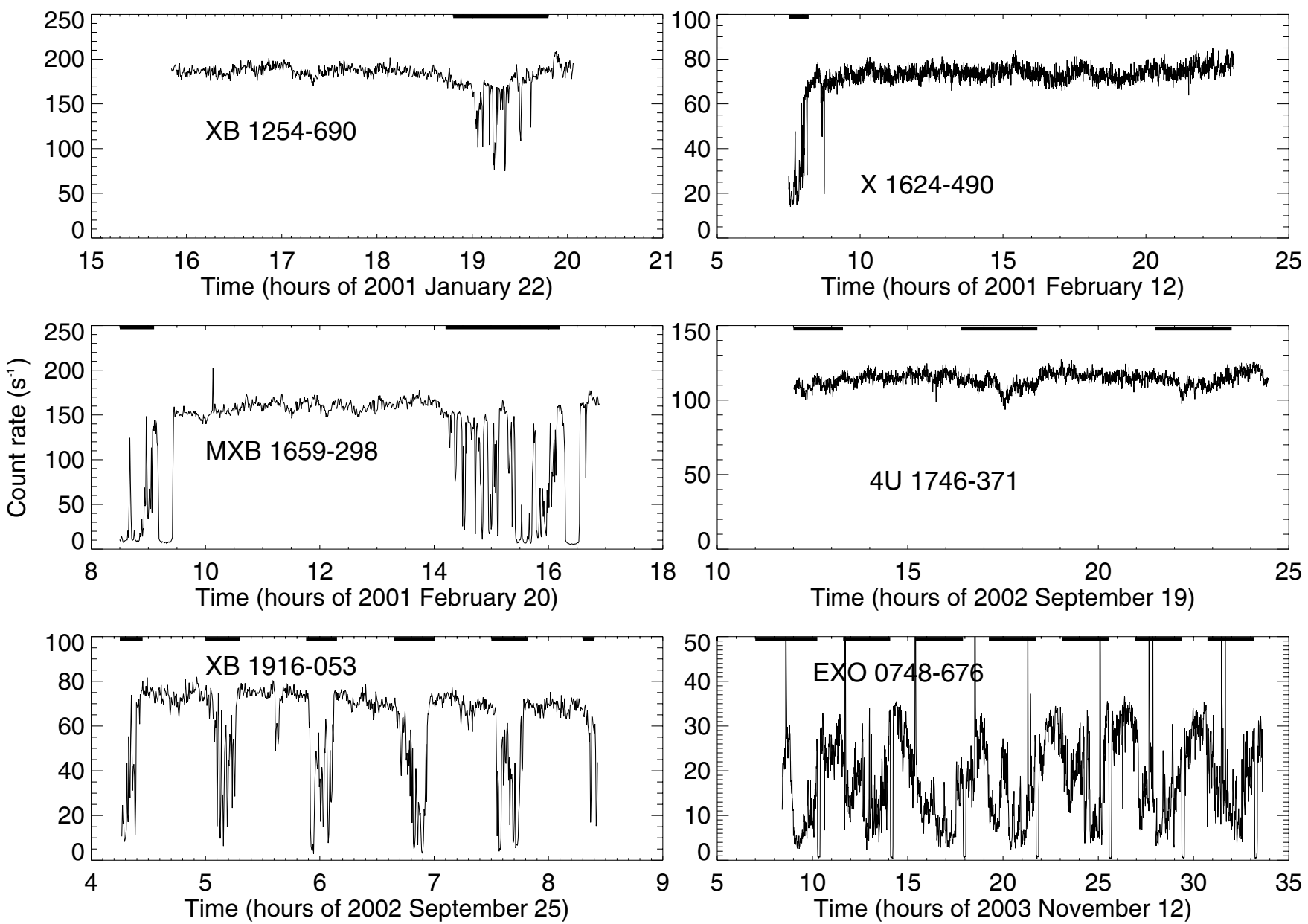

Fig. 1. EPIC pn 0.6-10 keV lightcurves. The binning is $20 \mathrm{~s}$ except for MXB 1659-298 and EXO0748-676, where it is $40 \mathrm{~s}$. The thick horizontal lines mark the intervals used to extract dip spectra.

Table 4. Dip intensity selection. Dip depth is given as a percentage of the $0.6-10 \mathrm{keV}$ pn persistent count rate (2-10 keV for X 1624-490). The accumulation time was $20 \mathrm{~s}$.

\begin{tabular}{cc}
\hline \hline Dip Level & Dip depth \\
\hline 1 & $<15 \%$ \\
2 & $15-30 \%$ \\
3 & $30-50 \%$ \\
4 & $30-65 \%$ \\
5 & $65-80 \%$ \\
\hline
\end{tabular}

and upper limits at $95 \%$ confidence. The $\chi^{2}$ values obtained from SPEX were calculated using the expected errors based on the model rather than the observed errors (see the SPEX user's manual). We quote all $E W \mathrm{~s}$ with positive values for both absorption and emission features. We rebinned the EPIC pn spectra to over-sample the full-width at half-maximum $(F W H M)$ of the energy resolution by a factor 3 , and to have a minimum of 25 counts per bin to allow the use of the $\chi^{2}$ statistic. To account for systematic effects we added quadratically a $2 \%$ uncertainty to each pn spectral bin.

In order to study the different sources in a systematic manner, we used for all the spectra the same continuum consisting of blackbody and power-law components modified by neutral absorption (the abs*(bb+pl) model in SPEX). For each source we fit simultaneously all the EPIC pn spectra of the persistent and dipping intervals with the continuum parameters tied together, while we allowed to vary all the other parameters. We included Gaussian emission profiles when emission features were evident near $1 \mathrm{keV}$ and/or $6 \mathrm{keV}$. The $1 \mathrm{keV}$ feature has been previously modeled either as an emission line, or as an edge, and its nature is unclear (e.g., Sidoli et al. 2001; Boirin \& Parmar 2003; Boirin et al. 2004, 2005). Examination of the spectral residuals reveals strong absorption features around $7 \mathrm{keV}$ for all the sources except EXO 0748-676 and 4U 1746-371. To account for these narrow features, we included absorption from a photo-ionized plasma (xabs in SPEX) in the spectral model.

The xabs model of SPEX treats, in a simplified manner, the absorption by a thin slab composed of different ions, located between the ionizing source and the observer. It assumes that the angle subtended by the slab as seen from the ionizing source is small. Therefore, emission from the slab and scattering by the slab of the ionizing source into the line-of-sight are neglected, and only absorption and scattering out of the line-of-sight by the slab are considered. The processes taken into account are the continuum and the line absorption by the 
Table 5. Ionizing continua used to pre-calculate grids of relative ionic column densities for fitting with the xabs model in SPEX (see text). The references are for the cutoff energies, $E_{c}$.

\begin{tabular}{lcccl}
\hline \hline LMXB & $\Gamma$ & $E_{\mathrm{c}}(\mathrm{keV})$ & Mission & Reference \\
\hline XB 1916-053 & 1.87 & 80 & BeppoSAX & Church et al. (1998) \\
XB 1323-619 & 1.96 & 44 & BeppoSAX & Bałucińska-Church et al. (1999) \\
EXO 0748-676 & 1.27 & 44 & BeppoSAX & Sidoli et al. (2005) \\
XB 1254-690 & 1.28 & 5.9 & R-XTE & Smale et al. (2002) \\
4U 1746-371 & 0.39 & 3.5 & BeppoSAX & Parmar et al. (1999) \\
MXB 1659-298 & 1.21 & 7.1 & BeppoSAX & Oosterbroek et al. (2001) \\
X 1624-490 & 2.00 & 12 & BeppoSAX & Bałucińska-Church et al. (2000) \\
\hline
\end{tabular}

ions and scattering out of the line-of-sight by the free electrons in the slab. Most continuum opacities are taken from Verner \& Yakovlev (1995), while line opacities and wavelengths for most ions are taken from Verner et al. (1996) (see the details and additional references in the SPEX user's manual). The relative column densities of the ions are coupled through a photoionization model. Using codes such as CLOUDY (Ferland 2003), and assuming a broad-band ionizing continuum from infra-red to hard X-rays, the ionic column densities of a photoionized slab can be pre-calculated for different values of $\xi$. SPEX reads in the grid of pre-calculated ionic column densities during the fitting process. In this paper, we use a grid of ionic column densities pre-calculated using CLOUDY and assuming that the ionizing continuum for each source may be represented by a cutoff power-law $\left(E^{-\Gamma} \exp \left(-E / E_{\mathrm{c}}\right)\right)$ with a power-law index, $\Gamma$, obtained from the fits to the EPIC pn persistent spectra with the cutoff energy, $E_{\mathrm{c}}$, fixed to the value measured with BeppoSAX or R-XTE (see Table 5).

The parameters of the xabs model that were allowed to vary are $N_{\mathrm{H}}^{\mathrm{xabs}}, \xi$ and $\sigma_{\mathrm{v}} . N_{\mathrm{H}}^{\mathrm{xabs}}$ is the equivalent hydrogen column density of the ionized absorber in units of atom $\mathrm{cm}^{-2} . \xi$ is the ionization parameter of the absorber, defined as $\xi=L / n_{\mathrm{e}} r^{2}$, where $L$ is the luminosity of the ionizing source, $n_{\mathrm{e}}$ the electron density of the plasma and $r$ the distance between the slab and the ionizing source. $\xi$ is expressed in units of erg $\mathrm{cm} \mathrm{s}^{-1}$, but we will omit the units when quoting $\log (\xi)$ values in this paper. $\sigma_{\mathrm{v}}$ is the turbulent velocity broadening of the absorber in $\mathrm{km} \mathrm{s}^{-1}$, defined as $\sigma_{\text {total }}^{2}=\sigma_{\mathrm{v}}^{2}+\sigma_{\text {thermal }}^{2}$, where $\sigma_{\text {total }}$ is the total width of a line and $\sigma_{\text {thermal }}$ the thermal contribution.

We present now the results of the fits for each of the sources individually before discussing the general properties of the absorbers in dipping LMXBs in Sect. 4.

\subsection{XB 1916-053}

Results of the fits of the XB 1916-053 persistent and dipping spectra to the abs*xabs*(pl+bb) + abs* (gau) SPEX model are shown in Fig. 2 and the best-fit parameter values are listed in Table 6 . The line and continuum changes can be primarily modeled by changes in the ionized absorber with $N_{\mathrm{H}}^{\mathrm{xabs}}$ increasing from $(4.2 \pm 0.5) \times 10^{22}$ to $(54 \pm 3) \times 10^{22}$ atom cm $\mathrm{cm}^{-2}$ while $\log (\xi)$ decreases from $3.05 \pm 0.04$ to $2.52_{-0.06}^{+0.02}$, with a smaller change in the amount of neutral absorption, $N_{\mathrm{H}}$, which increases from $(0.432 \pm 0.002) \times 10^{22}$ to $(0.89 \pm 0.07) \times$ $10^{22}$ atom $\mathrm{cm}^{-2}$. The $\sim 1 \mathrm{keV}$ feature is only visible in the
Dip 3 to Dip 5 spectra (see Table 4 for Dip definition). The strongest absorption lines in the persistent spectrum are from Fe XXV and Fe XXVI with EWs of $34 \mathrm{eV}$ and $21 \mathrm{eV}$, respectively. The Fe XXV and Fe XXVI Ly $\beta$ features are also predicted by the xabs model with $E W \mathrm{~s}$ of $7 \mathrm{eV}$ and $4 \mathrm{eV}$, respectively. During dipping, the $E W$ of the Fe XXVI line decreases and a feature is observed at an energy $6.6 \pm 0.1 \mathrm{keV}$, possibly originating from a blend of Fe XXV to Fe XIX lines. The best-fit value of $\log (\xi)$ is $3.05 \pm 0.04$ for the persistent spectrum. This is significantly lower than the value of 3.92 reported by Boirin et al. (2004). This difference may be explained by the fact that their result is based on the photo-ionized model by Kallman \& Bautista (2001) which assumes an ionizing continuum consisting of a power-law with $\Gamma=1$ while we use as ionizing continuum a cutoff power-law of $\Gamma=1.87$ and $E_{\mathrm{c}}=80 \mathrm{keV}$ (see Table 5).

\subsection{EXO $0748-676$}

Narrow X-ray absorption lines have been detected from EXO 0748-676 during X-ray bursts (Cottam et al. 2002), but such features have not been detected during persistent or dipping intervals. The $0.6-10 \mathrm{keV}$ pn persistent spectrum was first fit using a blackbody and a power-law model, together with photo-electric absorption by neutral material. The fit is unacceptable with a $\chi_{v}^{2}$ of $\sim 10$ for 235 degrees of freedom (d.o.f.) due mainly to the presence of strong soft excesses at $2 \mathrm{keV}$ and $\lesssim 1 \mathrm{keV}$. Even though no strong narrow absorption lines are evident, we investigated the possibility that the soft excesses result from the presence of an ionized absorber by fitting the persistent spectrum with the standard abs*xabs*(pl+bb) SPEX model. A minimum value of $1.1 \times 10^{21}$ atom $\mathrm{cm}^{-2}$ (Sidoli et al. 2005) was imposed on $N_{\mathrm{H}}$ to prevent this parameter going to unrealistically low values. The soft excesses were both well modeled by the ionized absorber, but the fit is still formally unacceptable with a $\chi_{v}^{2}$ of $\sim 3$ for 232 d.o.f. Examination of the residuals reveals narrow emission features at energies $\lesssim 2 \mathrm{keV}$, which were modeled by including two Gaussian emission lines at 0.57 , and $0.92 \mathrm{keV}$, the energies of inter-combination lines of O VII and Ne IX, respectively. The fit is now significantly improved with a $\chi_{v}^{2}$ of 1.4 for 225 d.o.f. Next we fit the persistent and dipping spectra together in the usual manner with the model abs*xabs*(pl+bb)+abs* (gau+gau). The best-fit parameters are given in Table 7 and the spectra are shown in Fig. 3. The changes in the continuum can be modeled 

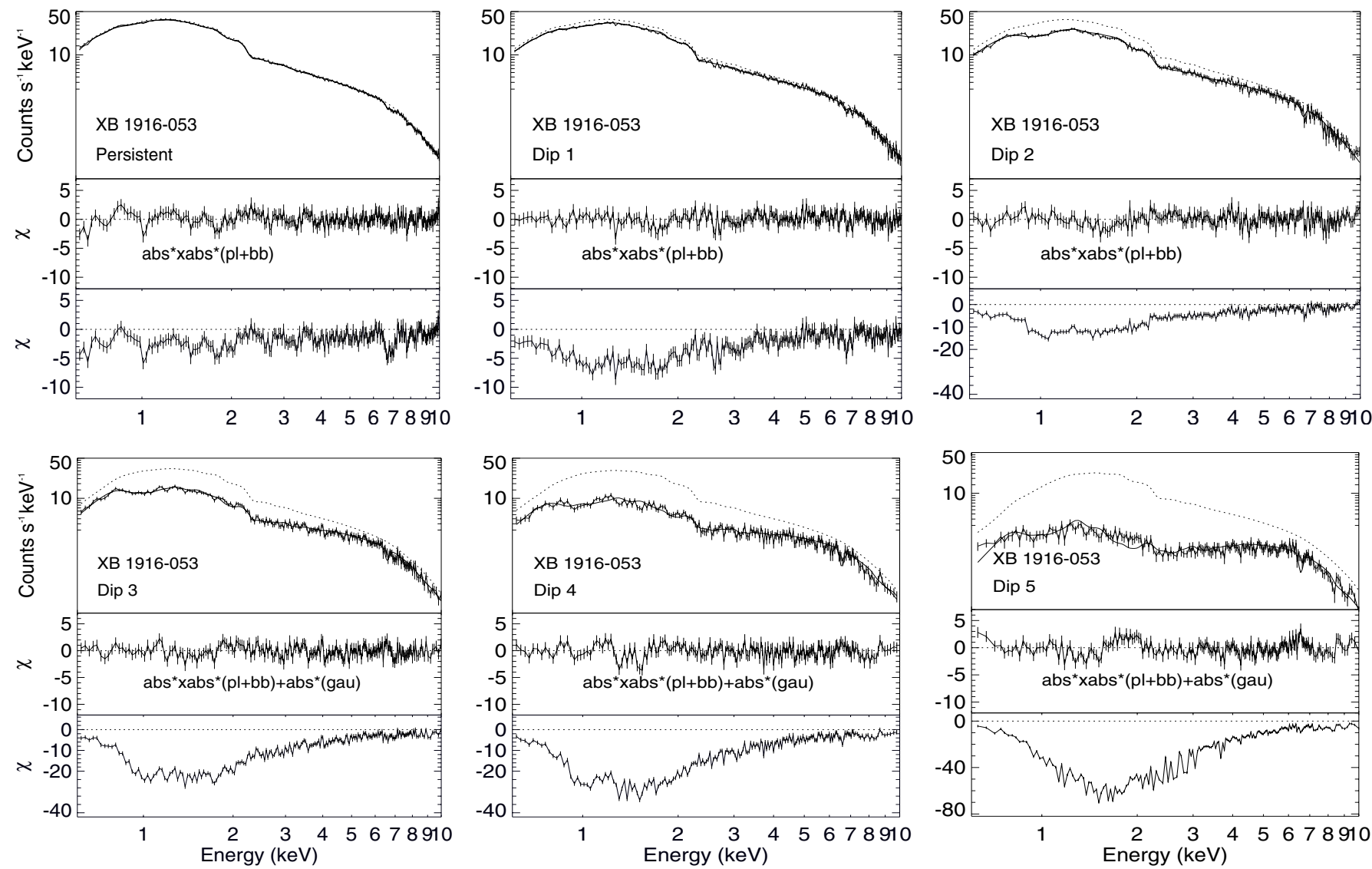

Fig. 2. XB 1916-053: EPIC pn persistent and dipping spectra fit with a power-law (pl) and a blackbody (bb) model, modified by absorption from neutral (abs) and ionized (xabs) material plus one narrow emission line (gau), modified by absorption from neutral material (abs) (see Table 6). The dotted lines show the models when the column of the ionized absorber is set to 0 . The middle panels show residuals in units of standard deviations from the above model. The lower panels show residuals when $N_{\mathrm{H}}^{\mathrm{xabs}}$ is set to 0 .

primarily by changes in the ionized absorber with a large increase in $N_{\mathrm{H}}^{\mathrm{xabs}}$ from $(3.5 \pm 0.2) \times 10^{22}$ atom $\mathrm{cm}^{-2}$ to $(15.5 \pm$ $0.5) \times 10^{22}$ atom $\mathrm{cm}^{-2}$ while $\log (\xi)$ decreases from $2.45 \pm 0.02$ to $2.26 \pm 0.03$. A smaller increase in the amount of neutral absorption from $0.11 \times 10^{22}$ to $\left(0.24_{-0.05}^{+0.09}\right) \times 10^{22}$ atom $\mathrm{cm}^{-2}$ is required. The ionized absorber is not well constrained due to the absence of strong individually resolved absorption features in the spectra. In contrast, strong absorption edges are evident, which are produced e.g., in the persistent emission, by O VIII and $\mathrm{Ne} \mathrm{X}$ at 0.87 and $1.36 \mathrm{keV}$ with optical depths of 0.39 and 0.09 , respectively.

\subsection{XB 1254-690}

The XMM-Newton lightcurve of XB 1254-690 shows evidence for enhanced background counting rates at the time of occurrence of the dip, with background peak count rates $\$ 10 \mathrm{~s}^{-1}$, compared to a source count rate of $90 \mathrm{~s}^{-1}$ during deepest dipping. For this reason Boirin \& Parmar (2003) did not examine the spectral changes during the dip seen from XB 1254-690. Since this is the only XMM-Newton observation of a dip from XB 1254-690, we included the dip data in our analysis. We caution that even though background subtraction is not critical for such a bright source, the spectral fits may be affected by the enhanced background. The best-fits of the persistent and dipping emission of XB 1254-690 to the abs*xabs*(pl+bb) + abs*(gau) SPEX model are shown in Fig. 4 and the parameter values given in Table 8 . The changes in the continuum can be modeled primarily by changes in the ionized absorber with $N_{\mathrm{H}}^{\mathrm{xabs}}$ increasing from $(8.4 \pm 0.3) \times 10^{22}$ to $(47 \pm 3) \times 10^{22}$ atom $\mathrm{cm}^{-2}$ while $\log (\xi)$ decreases from $4.3 \pm 0.1$ to $2.94 \pm 0.05$ with a smaller increase in the amount of neutral absorption, from $(0.346 \pm 0.002) \times 10^{22}$ to $(0.39 \pm$ $0.01) \times 10^{22}$ atom $\mathrm{cm}^{-2}$.

The deepest line in the persistent spectrum is identified with Fe XXVI 1s-2p Ly $\alpha$ with an $E W$ of $32 \mathrm{eV}$ and is superposed on a broad emission feature with an energy of $6.8 \pm 0.1 \mathrm{keV}$

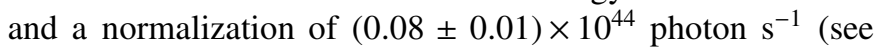
Fig. 4). The second strongest line is identified with a mixture of Fe XXVI 1s-2p Ly $\beta$ and Ni XXVIII with an $E W$ of $8 \mathrm{eV}$. During dipping, the $E W$ of the Fe XXVI line decreases and lines of lessionized species of Fe, such as Fe XXV, appear.

\section{4. $4 U 1746-371$}

No narrow X-ray absorption features have been reported for this source. The observation starts during a dip and two more complete dips are present in the lightcurve (Fig. 1). The dips are very shallow (85\% of the persistent emission remains) and difficult to identify. We find a dip recurrence period 
Table 6. XB 1916-053: best-fits to the EPIC pn persistent and 5 dip spectra using the abs*xabs*(pl+bb)+abs* (gau) model. The components of the continuum $(\mathrm{pl}+\mathrm{bb})$ are identical for all the spectra and only the $N_{\mathrm{H}}$ of the neutral absorber, the emission line parameters and $N_{\mathrm{H}}^{\mathrm{xabs}}, \sigma_{v}$ and $\xi$ of the ionized absorber are individually fit for each spectrum. $F_{\mathrm{X}}$ is the $0.6-10 \mathrm{keV}$ absorbed flux. The $F W H M$ of the Gaussian emission line is constrained to be $\leq 0.2 \mathrm{keV}$.

\begin{tabular}{|c|c|c|c|c|c|c|}
\hline & Persistent & Dip 1 & Dip 2 & Dip 3 & Dip 4 & Dip 5 \\
\hline & & & & & & \\
\hline \multicolumn{7}{|l|}{ Parameter } \\
\hline & & & & & & \\
\hline$\Gamma$ & \multicolumn{6}{|c|}{$2.25 \pm 0.03$} \\
\hline \multicolumn{3}{|l|}{$k_{\mathrm{pl}}\left(10^{44}\right.$ ph. $\left.\mathrm{s}^{-1} \mathrm{keV}^{-1}\right)$} & \multicolumn{2}{|c|}{$10.0 \pm 0.2$} & & \\
\hline 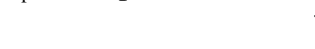 & & & & & & \\
\hline$k T_{\mathrm{bb}}(\mathrm{keV})$ & \multicolumn{6}{|c|}{$1.95 \pm 0.04$} \\
\hline$k_{\mathrm{bb}}\left(10^{11} \mathrm{~cm}^{2}\right)$ & \multicolumn{6}{|c|}{$0.65 \pm 0.08$} \\
\hline & & & & & & \\
\hline$N_{\mathrm{H}}^{\mathrm{abs}}\left(10^{22} \mathrm{~cm}^{-2}\right)$ & $0.432 \pm 0.002$ & $0.444 \pm 0.004$ & $0.450 \pm 0.006$ & $0.54 \pm 0.02$ & $0.60 \pm 0.02$ & $0.89 \pm 0.07$ \\
\hline$N_{\mathrm{H}}^{\mathrm{xabs}}\left(10^{22} \mathrm{~cm}^{-2}\right)$ & $4.2 \pm 0.5$ & $9 \pm 2$ & $16 \pm 2$ & $22 \pm 2$ & $28 \pm 2$ & $54 \pm 3$ \\
\hline $\log (\xi)\left(\mathrm{erg} \mathrm{cm} \mathrm{s}^{-1}\right)$ & $3.05 \pm 0.04$ & $2.84 \pm 0.04$ & $2.75 \pm 0.02$ & $2.62_{-0.06}^{+0.02}$ & $2.55 \pm 0.04$ & $2.52_{-0.06}^{+0.02}$ \\
\hline \multirow[t]{2}{*}{$\sigma_{\mathrm{v}}\left(\mathrm{km} \mathrm{s}^{-1}\right)$} & $2300_{-1700}^{+2100}$ & $50 \pm 30$ & $90 \pm 25$ & $120_{-20}^{+40}$ & $220_{-90}^{+80}$ & $160_{-40}^{+100}$ \\
\hline & & & & & & \\
\hline$E_{\text {gau }}(\mathrm{keV})$ & & & & $0.89 \pm 0.03$ & $0.92 \pm 0.04$ & $0.88 \pm 0.04$ \\
\hline$F W H M(\mathrm{keV})$ & & & & 0.2 & 0.2 & 0.2 \\
\hline$k_{\text {gau }}\left(10^{44} \mathrm{ph} \mathrm{s}^{-1}\right)$ & & & & $0.6 \pm 0.1$ & $0.6 \pm 0.1$ & $0.5 \pm 0.3$ \\
\hline $\begin{array}{l}F_{\mathrm{X}}\left(10^{-10} \mathrm{erg} \mathrm{cm}^{-2} \mathrm{~s}^{-1}\right) \\
\chi_{\nu}^{2} \text { (d.o.f.) }\end{array}$ & 2.6 & 2.4 & $\begin{array}{l}2.0 \\
1.30(12\end{array}$ & 1.5 & 1.2 & 0.7 \\
\hline Exposure (ks) & 9.1 & 2.1 & 1.1 & 0.9 & 0.6 & 0.8 \\
\hline
\end{tabular}

of $\sim 5.1 \mathrm{~h}$, in agreement with the value of $5.16 \pm 0.01 \mathrm{~h}$ reported in Bałucińska-Church et al. (2004). The observation was divided into persistent and dip spectra which were fit separately using an absorbed power-law and blackbody wabs $(b b+p l)$ model. The fit quality is acceptable with $\chi_{\nu}^{2} \mathrm{~s}$ of 1.32 and 1.23 for 229 d.o.f for persistent and dip spectra, respectively. Examination of the residuals reveals a broad emission feature at an energy of $\sim 6.4 \mathrm{keV}$ in both spectra. This feature was modeled as a Gaussian emission line with energies of $6.45_{-0.29}^{+0.25} \mathrm{keV}$ and $6.1_{-0.2}^{+0.3} \mathrm{keV}$, widths $(\sigma)$ of $0.85 \mathrm{keV}$ and $E W \mathrm{~s}$ of $181 \pm 55 \mathrm{eV}$ and $175 \pm 60 \mathrm{eV}$ for the persistent and dip spectra, respectively. This results in $\chi_{v}^{2} \mathrm{~s}$ of 1.23 and 1.14 for 226 d.o.f. The F-statistic values of 6.33 and 7.38 indicate that the probability of such a decrease occurring by chance is $4 \times 10^{-4}$ and $1 \times 10^{-4}$. A Gaussian absorption line with an energy of $6.9 \pm 0.1 \mathrm{keV}$, a width $(\sigma)$ of $<0.80 \mathrm{keV}$ and an $E W$ of $30 \pm 15 \mathrm{eV}$ is the best-fit to the absorption feature in the persistent spectrum. This results in a $\chi_{v}^{2}$ of 1.21 for 223 d.o.f. The F-statistic value of 2.19 indicates that the probability of such a decrease occurring by chance is only 0.09 . Table 9 gives the best-fit parameters and the residuals are shown in Fig. 5.

The Fe XXVI narrow absorption feature indicates the presence of a highly-ionized absorber. The upper limit $E W$ to a narrow feature at the energy of Fe XXV is $<5 \mathrm{eV}$. We then fit the persistent and dip spectra simultaneously with the abs*xabs*(pl+bb)+abs*(gau+gau) model of SPEX. Unfortunately, due to the shallowness of the Fe XXVI feature the highly-ionized absorber is not well constrained and the best-fit values of $N_{\mathrm{H}}^{\mathrm{xabs}}$ are highly correlated with variations in the normalization of the continuum.

\section{5. $M X B$ 1659-298}

The best-fit parameters of the MXB 1659-298 persistent and dipping emission to the $a b s^{*} \times a b s *(p l+b b)+$ abs* (gau+gau) SPEX model are shown in Table 10 and the spectra and residuals in Fig. 6. The changes in the continuum can be modeled primarily by a change in the ionized absorber with $N_{\mathrm{H}}^{\mathrm{xabs}}$ increasing from $(11.1 \pm 0.6) \times 10^{22}$ to $(53 \pm 3) \times 10^{22}$ atom $\mathrm{cm}^{-2}$ while $\log (\xi)$ decreases from $3.8 \pm$ 0.1 to $2.42_{-0.06}^{+0.02}$ with a smaller change in the amount of neutral absorption, $N_{\mathrm{H}}$, which increases from $(0.306 \pm 0.003) \times 10^{22}$ to $(0.71 \pm 0.04) \times 10^{22}$ atom $\mathrm{cm}^{-2}$.

The $\sim 1 \mathrm{keV}$ feature is not visible in the Dip 1 and 2 spectra. The $\sim 7 \mathrm{keV}$ broad emission feature is only present in the persistent spectrum. The strongest absorption lines in the persistent spectrum are identified with Fe XXV and Fe XXVI with $E W$ s of $32 \mathrm{eV}$ and $48 \mathrm{eV}$, respectively (see Fig. 6). Fe XXV and Fe XXVI Ly $\beta$ absorption features are also predicted by the xabs model with $E W$ s of $9 \mathrm{eV}$ and $12 \mathrm{eV}$, respectively. During dipping, the $E W$ of the Fe XXVI line decreases and the feature observed at an energy of $\sim 6.60 \mathrm{keV}$ is identified with a blend of Fe XXV to Fe XIX lines. 
Table 7. EXO 0748-676: best-fits to the pn persistent and 5 dip spectra using the abs*xabs*(pl+bb)+abs*(gau+gau) model. The continuum parameters $(\mathrm{pl}+\mathrm{bb})$ are the same for all spectra and only the $N_{\mathrm{H}}$ of the neutral absorber, the line parameters, and $N_{\mathrm{H}}^{\mathrm{xabs}}, \sigma_{v}$ and $\xi$ of the ionized absorber are fit for each spectrum. $F_{\mathrm{X}}$ is the $0.6-10 \mathrm{keV}$ absorbed flux. $N_{\mathrm{H}}$ is constrained to be $\geq 1.1 \times 10^{21}$ atom $\mathrm{cm}^{-2}$ (Sidoli et al. 2005) and the FWHMs of the emission lines are constrained to be $\leq 0.20 \mathrm{keV}$.

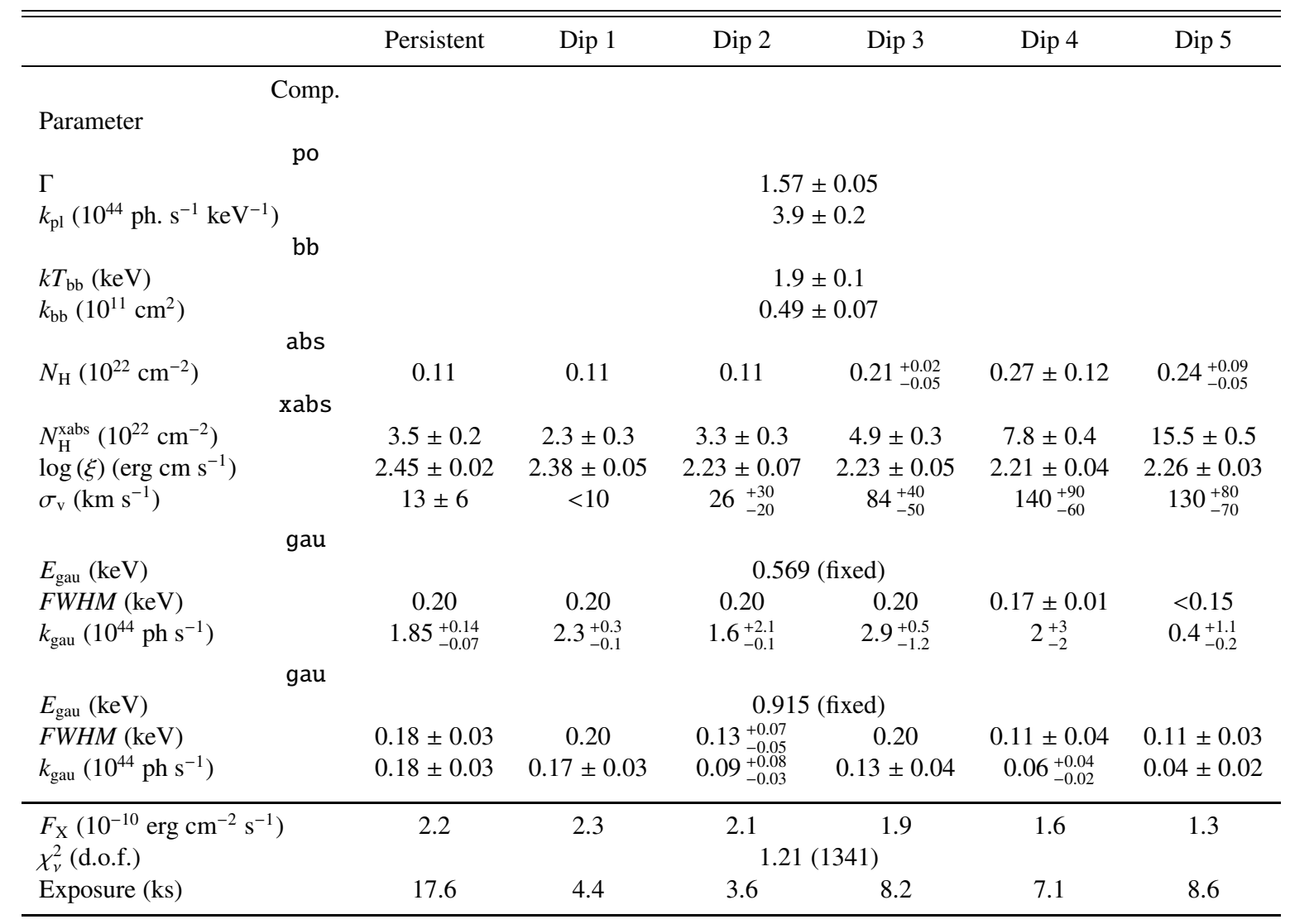

From all the studied sources MXB 1659-298 shows the deepest Fe XXV and Fe XXVI absorption lines and is the only dipping LMXB which shows strong narrow absorption lines in the RGS (Sidoli et al. 2001). The latter features are produced by O VIII $1 \mathrm{~s}-2 \mathrm{p}, 1 \mathrm{~s}-3 \mathrm{p}, 1 \mathrm{~s}-4 \mathrm{p}$ and $\mathrm{Ne} \mathrm{X} 1 \mathrm{~s}-2 \mathrm{p}$, at $18.95_{-0.01}^{+0.02}$, $16.00_{-0.01}^{+0.02}, 15.21 \pm 0.01$ and $12.15_{-0.01}^{+0.02} \AA$ and indicate the presence of less ionized material than that responsible for the Fe XXV and Fe XXVI absorption features. Thus, the absorbing material may have a range of ionization states: the material closer to the compact object may be more ionized and produce the Fe XXV and Fe XXVI lines while the one farther from the compact object may be less ionized and produce the $\mathrm{O}$ VIII and Ne X lines. The latter features are however not predicted by the xabs model. This is probably because when fitting an absorber with only one ionization state, the Fe XXV and Fe XXVI lines dominate over the $\mathrm{O}$ VIII and $\mathrm{NeX}$ lines due to their larger $E W \mathrm{~s}$. We next performed a fit to the persistent RGS first order spectra with the SPEX abs*xabs*(p1) model. The RGS dipping spectra have too few counts for spectral fitting. We find $N_{\mathrm{H}}=(0.287 \pm 0.007) \times 10^{22}$ atom $\mathrm{cm}^{-2}$, $N_{\mathrm{H}}^{\mathrm{xabs}}=\left(3_{-2}^{+5}\right) \times 10^{22}$ atom $\mathrm{cm}^{-2}, \log (\xi)=3.1 \pm 0.3$ and $\sigma_{\mathrm{v}}=$ $190{ }_{-100}^{+180} \mathrm{~km} \mathrm{~s}^{-1}$. The uncertainties in these parameters are large in comparison with those found for the pn fit. Despite the uncertainties, a less ionized absorber is required by the RGS data compared to the pn. Narrow absorption features of
O VIII $1 \mathrm{~s}-2 \mathrm{p}, 1 \mathrm{~s}-3 \mathrm{p}, 1 \mathrm{~s}-4 \mathrm{p}$ and $\mathrm{Ne} \mathrm{X} 1 \mathrm{~s}-2 \mathrm{p}$ are predicted by the xabs model with $E W$ s of 2.2, 0.7, 0.3 and $2.0 \mathrm{eV}$, respectively. These values are slightly lower than those found by Sidoli et al. (2001) of $2.6 \pm 0.4,1.3 \pm 0.4,1.6 \pm 0.4$ and $2.1 \pm 0.4 \mathrm{eV}$ for the corresponding lines. This difference may be explained by our use of a complete photo-ionized absorber rather than individual spectral features. Thus, the assumption of a range of different ionization states for the absorber seems correct. However, when we added a second absorber to our model with $\log (\xi)$ fixed to the RGS value and performed a fit to the pn persistent spectrum, the $\chi^{2}$ of the fit did not improve significantly. This suggests that the nature of the absorber is even more complex. Alternatively, the $\mathrm{O}$ VIII and $\mathrm{Ne} \mathrm{X}$ features may be too weak in the pn spectrum to constrain properly the second ionized absorber, since such features have been reported only in the RGS data, which has a resolution $(E / \Delta E)$ of $\sim 300$ compared to $\sim 12$ for the pn at $1 \mathrm{keV}$.

\section{6. $\times 1624-490$}

The radial intensity profile of the source obtained using the EPIC pn image revealed a constant excess in intensity at large radii $\left(>30^{\prime \prime}\right)$ during both persistent and dipping intervals, demonstrating the presence of a dust-scattering halo, first detected from this source by Angelini et al. (1997). The galactic 

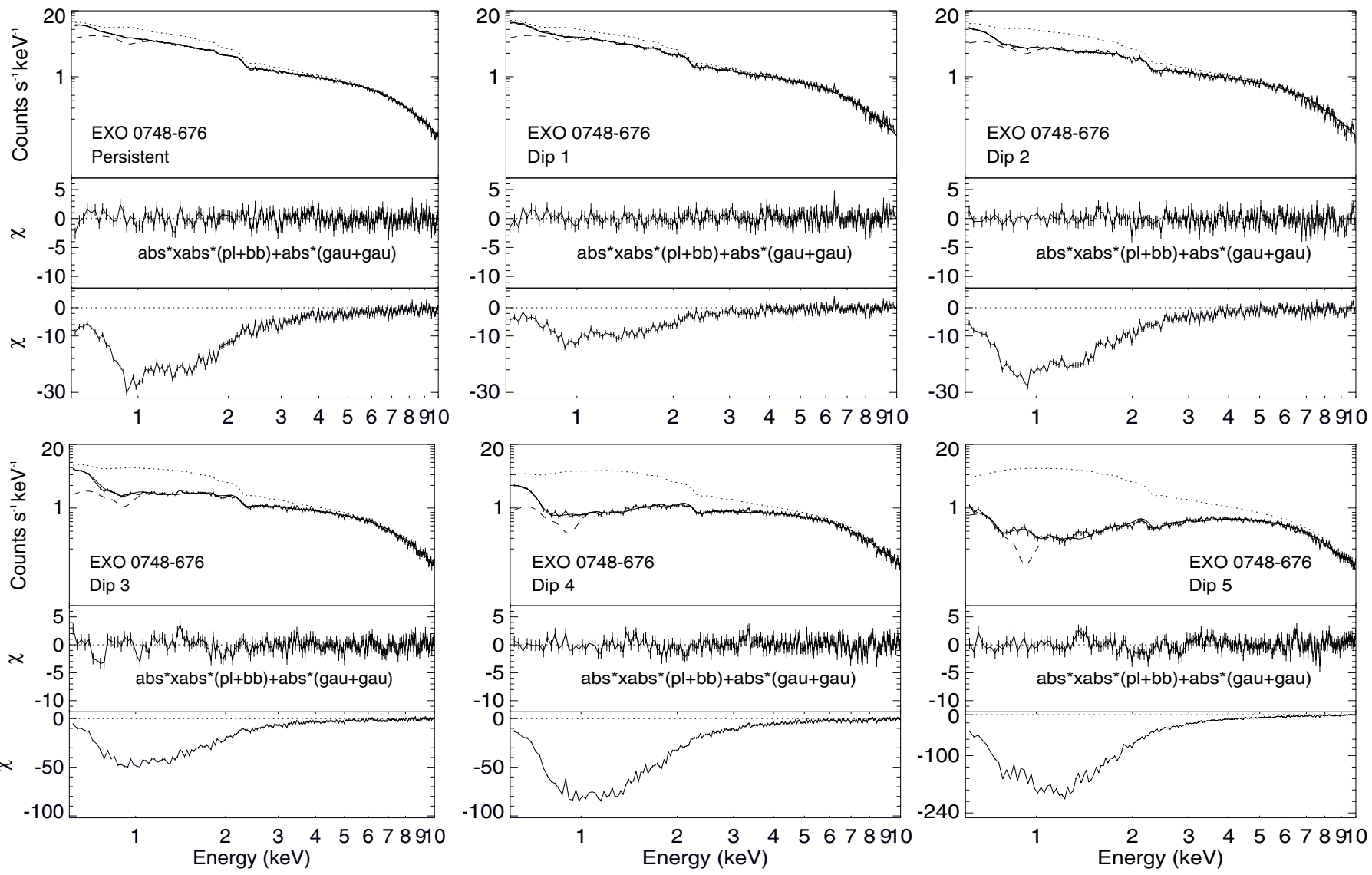

Fig. 3. EXO 0748-676: EPIC pn persistent and 5 dipping spectra fit with a power-law (pl) and blackbody (bb) modified by absorption from neutral (abs) and ionized (xabs) material with 2 narrow emission lines (gau) (see Table 7). The dotted lines show the models when the column of the ionized absorber is set to 0 . The dashed lines show the models when the normalizations of the Gaussian emission features are set to 0 . The middle panels show the residuals in units of standard deviations from the above model. The lower panels show the residuals when $N_{\mathrm{H}}^{\mathrm{xab}}$ is set to 0 .

column density of X 1624-490 is high $\left(\sim 8 \times 10^{22}\right.$ atom $\mathrm{cm}^{-2}$, Parmar et al. 2002), so that a dust scattered halo is expected. To model this we used $a b s_{1} * x^{2} a b s e^{-\tau}(b b+p l)+$ $\mathrm{abs}_{2} *\left(1-\mathrm{e}^{-\tau}\right) *(\mathrm{bb}+\mathrm{pl})$. The first component represents the contribution of the source reduced by a factor $\mathrm{e}^{-\tau}$ (component etau in SPEX), corresponding to scattering out of the line of sight. The scattering into the line of sight due to the halo is represented by the second component and is expected to be the same for persistent and dipping spectra. $\tau$ is defined as $\tau_{1} E^{-2}$, where $\tau_{1}$ is the optical depth at $1 \mathrm{keV}$ and $E^{-2}$ is the theoretical energy dependence of the dust scattering cross-section. Unfortunately, the X 1624-490 persistent spectrum is strongly piled-up and counts from the inner circle of $20^{\prime \prime}$ radius had to be excluded. Thus, the resultant spectra contain a larger fraction of the halo component. For this reason we decided to use only the dipping spectra for the fit, since then the inner $20^{\prime \prime}$ radius circle does not have to be excluded and there is a larger contribution from direct source emission. We imposed a lower limit of 1.90 to the power-law photon index, $\Gamma$, to prevent it becoming negative without significantly changing the $\chi^{2}$ of the fit. The value of 1.90 was taken from Parmar et al. (2002) (their value of $\Gamma=2.02 \pm 0.12$ gives a minimum value of 1.90 ). The $N_{\mathrm{H}}$ of the neutral absorber for the Dip 1 spectrum was coupled to the value of $N_{\mathrm{H}}$ for the halo, assuming that the source must be at least as absorbed as the halo. The best-fit parameters for the dipping emission of X 1624-490 are shown in Table 11 and the spectra and residuals in Fig. 7. Significant changes in both the ionized and neutral absorbers are required to obtain satisfactory fits with $N_{\mathrm{H}}$ increasing from $(10.7 \pm 0.5) \times 10^{22}$ to $\left(59_{-3}^{+6}\right) \times 10^{22}$ atom $\mathrm{cm}^{-2}$ and $N_{\mathrm{H}}^{\mathrm{xabs}}$ from $(13 \pm 2) \times 10^{22}$ to $(68 \pm 9) \times 10^{22}$ atom $\mathrm{cm}^{-2}$. The value of $\log (\xi)$ decreases from $3.6 \pm 0.2$ to $3.0 \pm 0.2$ between Dip 1 and Dip 4. For Dip 5 the value of $\log (\xi)$ is not well constrained and we can only give a lower limit of $\geq 3.3$. Below $\sim 5 \mathrm{keV}$ the contribution of the halo (dashed line) dominates during the deepest dip stages. The optical depth of the dust, $\tau_{1}=1.8 \pm 0.2$, is consistent with that found by Bałucińska-Church et al. (2000) of $2.4 \pm 0.4$.

\section{Ionized absorber properties}

Examination of the spectral fit results (Tables 6 to 11) shows that we are able to successfully account for the complex changes in the $0.6-10 \mathrm{keV}$ continuum and absorption lines during dips from the LMXBs studied here (with the exception of $4 \mathrm{U} 1746-371$ where the dips are very shallow) by large increases in the column density, $N_{\mathrm{H}}^{\mathrm{xabs}}$, and decreases in the amount of ionization, $\xi$, of a highly-ionized absorber, together with much smaller increases in the $N_{\mathrm{H}}$ of a neutral absorber (for X 1624-490 the increase in the column densities of the neutral 

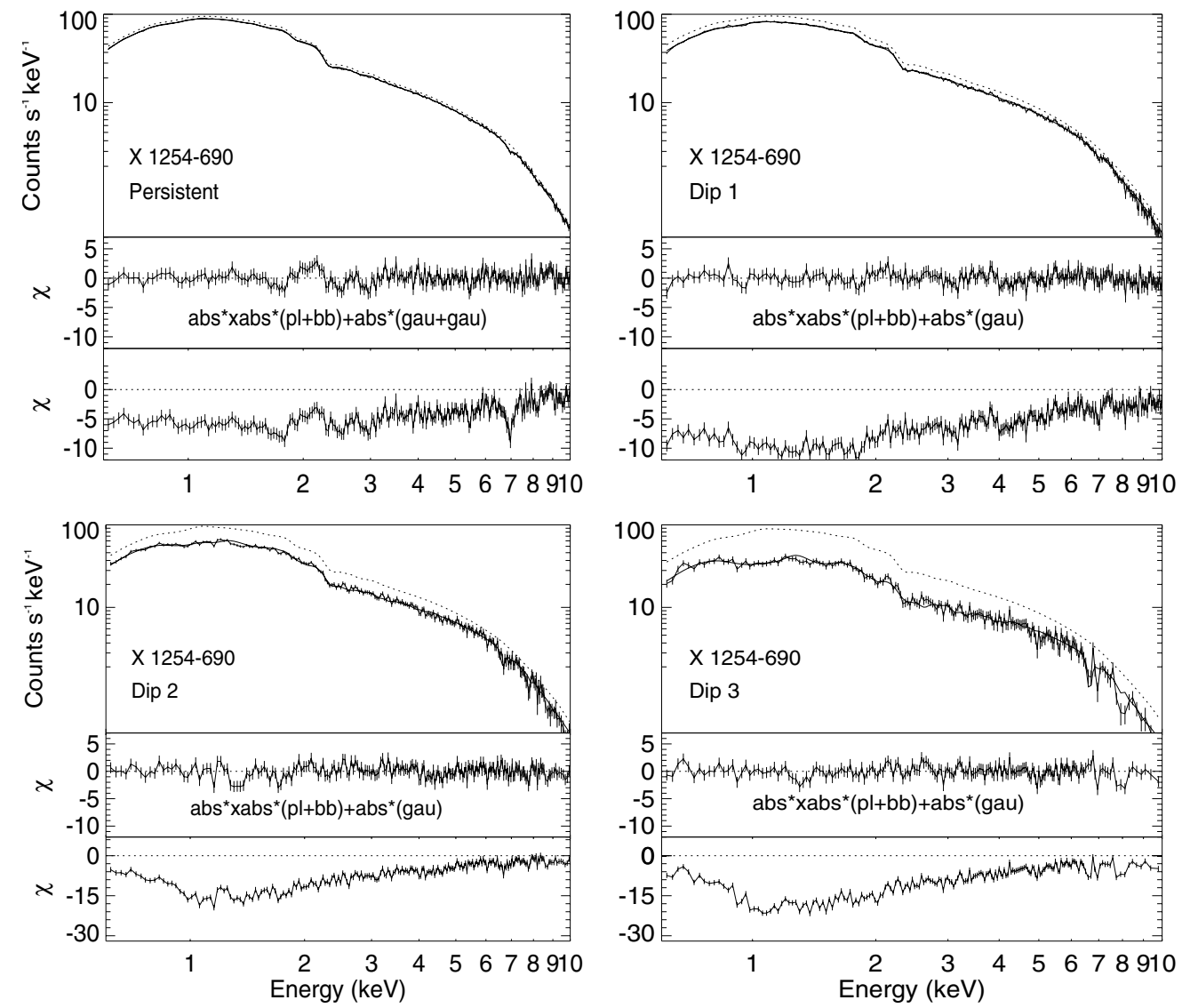

Fig. 4. XB 1254-690: EPIC pn persistent and 3 dipping spectra fit with a power-law (pl) and blackbody model (bb), modified by absorption from neutral (abs) and ionized (xabs) material and 2 narrow emission lines (gau), modified by absorption from neutral material (abs) (see Table 8). The dotted lines show the models when the column of the ionized absorber is set to 0 . The middle panels show the residuals in units of standard deviations from the above model. The lower panels show residuals when $N_{\mathrm{H}}^{\mathrm{xabs}}$ is set to 0 .

and ionized absorbers are comparable). The EWs of the lines predicted by xabs are consistent with previous measurements for the persistent emission (see Table 2).

During the persistent intervals, the values of $\sigma_{\mathrm{v}}$ are poorly constrained, except for EXO 0748-676 (see Tables 6 to 11). This is evidence for the absorption lines being unsaturated, i.e. on the linear part of the curve of growth, where the $E W$ increases linearly with the column density, independently of the velocity broadening. In contrast, in the deep dipping spectra, the values of $N_{\mathrm{H}}^{\mathrm{xabs}}$ tend to be poorly constrained whereas $\sigma_{\mathrm{v}}$ is well constrained. This indicates that the lines are probably saturated and their EWs do not strongly depend on the column density, but rather increase with velocity broadening. EXO 0748-676 is very likely in a saturated regime already in the persistent emission. This is indicated by the well constrained $\sigma_{\mathrm{v}}$ value together with the presence of absorption edges stronger than the narrow absorption features.

We tried to model the spectral changes during dipping emission of the studied LMXBs by changes only in the highlyionized absorbers present in these systems, but we could not obtain successful fits when the deep dipping spectra are included, unless the $N_{\mathrm{H}}$ of the neutral absorber is allowed to vary. As an example, we could adequately model the XB 1916-053 persistent and dipping spectra when the $N_{\mathrm{H}}$ was fixed for the persistent, Dip 1 and Dip 2 spectra $\left(\chi_{v}^{2}=1.36\right.$ for 1266 d.o.f. $)$, but the fit quality became unacceptable when we fixed the $N_{\mathrm{H}}$ of the deeper (Dip 3 to Dip 5) spectra, with structured residuals evident $\lesssim 2 \mathrm{keV}$ and a $\chi_{v}^{2}=2.44$ for 1269 d.o.f. This means that the spectral evolution requires changes in both the ionized and the neutral absorbers.

For all the sources, the strongest absorption lines in the persistent spectra are the FeXXV and Fe XXVI 1s-2p transitions except for XB 1254-690, where the absorber is the most highly ionized with $\log (\xi)=4.2 \pm 0.2$ and only the Ly $\alpha$ and $\beta$ Fe XXVI absorption features are evident. EXO 0748-676 and $4 \mathrm{U} 1746-371$ do not exhibit strong absorption lines. Our analysis indicates that the EXO 0748-676 absorber is significantly less ionized than in the other LMXBs studied with $\log (\xi)=2.45 \pm 0.02$. This could indicate that the intervals identified as "persistent" in Fig. 1 are not actually dip-free, and the source may be continuously dipping. This idea is supported by the large fraction of time that the source appears to be dipping, the well constrained value of $\sigma_{\mathrm{v}}$ during the "persistent" interval (Table 7) which may indicate that the source is already in the saturated line regime reached by the other sources only during dipping (see above), and by the low luminosity when compared to the other sources with similar values of $P_{\text {orb }}$ (Table 1$)$. In contrast, for $4 \mathrm{U}$ 1746-371 the non-detection of an Fe XXV absorption feature and the evidence of one from Fe XXVI indicate a highly-ionized absorber, similar to the one in XB 1254-690. 
Table 8. XB 1254-690: best-fits to the EPIC pn persistent and 3 dip spectra using the abs*xabs*(pl+bb)+abs*(gau+gau) model. The parameters for the continuum components $(\mathrm{pl}+\mathrm{bb})$ are the same for all the spectra and only the $N_{\mathrm{H}}$ of the neutral absorber, the line parameters, and $N_{\mathrm{H}}^{\mathrm{xabs}}, \sigma_{v}$ and $\xi$ of the ionized absorber are individually fit for each spectrum. $F_{\mathrm{X}}$ is the $0.6-10 \mathrm{keV}$ absorbed flux. The $F W H M$ of the $6.8 \mathrm{keV}$ Gaussian emission line is constrained to be $\leq 2.0 \mathrm{keV}$. The $F W H M \mathrm{~s}$ of the $\sim 1 \mathrm{keV}$ Gaussian emission lines are constrained to be $\leq 0.2 \mathrm{keV}$.

\begin{tabular}{|c|c|c|c|c|}
\hline & Persistent & Dip 1 & Dip 2 & Dip 3 \\
\hline & & & & \\
\hline \multicolumn{5}{|l|}{ Parameter } \\
\hline & & & & \\
\hline$\Gamma$ & \multicolumn{4}{|c|}{$2.09 \pm 0.02$} \\
\hline$k_{\mathrm{pl}}\left(10^{44}\right.$ ph. $\left.\mathrm{s}^{-1} \mathrm{keV}^{-1}\right)$ & \multicolumn{4}{|c|}{$19.4_{-0.5}^{+0.8}$} \\
\hline & & & & \\
\hline$k T_{\mathrm{bb}}(\mathrm{keV})$ & \multicolumn{4}{|c|}{$1.09 \pm 0.02$} \\
\hline \multirow[t]{2}{*}{$k_{\mathrm{bb}}\left(10^{11} \mathrm{~cm}^{2}\right)$} & \multicolumn{4}{|c|}{$16.1 \pm 0.7$} \\
\hline & & & & \\
\hline$N_{\mathrm{H}}\left(10^{22} \mathrm{~cm}^{-2}\right)$ & $0.346 \pm 0.002$ & $0.340 \pm 0.003$ & $0.350 \pm 0.006$ & $0.39 \pm 0.01$ \\
\hline$N_{\mathrm{H}}^{\mathrm{xabs}}\left(10^{22} \mathrm{~cm}^{-2}\right)$ & $8.4 \pm 0.3$ & $18.6 \pm 0.8$ & $25 \pm 2$ & $47 \pm 3$ \\
\hline $\log (\xi)\left(\mathrm{erg} \mathrm{cm} \mathrm{s}^{-1}\right)$ & $4.3 \pm 0.1$ & $3.9 \pm 0.3$ & $2.98_{-0.03}^{+0.06}$ & $2.94 \pm 0.05$ \\
\hline \multirow[t]{2}{*}{$\sigma_{\mathrm{v}}\left(\mathrm{km} \mathrm{s}^{-1}\right)$} & $2800 \pm 1900$ & $70 \stackrel{+70}{+50}$ & $130 \pm 35$ & $350_{-80}^{+110}$ \\
\hline & & & & \\
\hline$E_{\text {gau }}(\mathrm{keV})$ & $6.8 \pm 0.1$ & & & \\
\hline$F W H M(\mathrm{keV})$ & 2.0 & & & \\
\hline \multirow[t]{2}{*}{$k_{\text {gau }}\left(10^{44} \mathrm{ph} \mathrm{s}^{-1}\right)$} & $0.08 \pm 0.01$ & & & \\
\hline & & & & \\
\hline$E_{\text {gau }}(\mathrm{keV})$ & $1.00 \pm 0.02$ & $0.99 \pm 0.04$ & $1.07 \pm 0.03$ & $1.06 \pm 0.05$ \\
\hline$F W H M(\mathrm{keV})$ & 0.2 & 0.2 & $<0.2$ & $<0.2$ \\
\hline$k_{\text {gau }}\left(10^{44} \mathrm{ph} \mathrm{s}^{-1}\right)$ & $0.28 \pm 0.06$ & $0.22 \pm 0.07$ & $0.2_{-0.1}^{+0.2}$ & $0.2_{-0.1}^{+0.2}$ \\
\hline$F_{\mathrm{X}}\left(10^{-10} \mathrm{erg} \mathrm{cm}^{-2} \mathrm{~s}^{-1}\right)$ & 6.4 & 5.8 & 4.8 & 3.4 \\
\hline$\chi_{v}^{2}$ (d.o.f. $)$ & \multicolumn{4}{|c|}{$1.23(846)$} \\
\hline Exposure (ks) & 11.5 & 2.7 & 0.6 & 0.2 \\
\hline
\end{tabular}

Table 9. Best-fit spectral parameters for the persistent $4 \mathrm{U} 1746-371$ emission with the wabs $(\mathrm{bb}+\mathrm{pl}+\mathrm{gau}+\mathrm{gau})$ model. The $\sigma$ of the $\sim 6.4 \mathrm{keV}$ Gaussian emission line is constrained to be $\leq 0.85 \mathrm{keV}$.

\begin{tabular}{llc}
\hline \hline Comp. & Parameter & Value \\
\hline wabs & $N_{\mathrm{H}}\left(10^{22} \mathrm{~cm}^{-2}\right)$ & $0.37 \pm 0.01$ \\
$\mathrm{pl}$ & $\Gamma$ & $1.69 \pm 0.03$ \\
& $k_{\mathrm{pl}}\left(\right.$ photon $\left.\mathrm{keV}^{-1} \mathrm{~cm}^{-2} \mathrm{~s}^{-1}\right)$ & $0.115 \pm 0.003$ \\
$\mathrm{bb}$ & $k T_{\mathrm{bb}}(\mathrm{keV})$ & $1.28 \pm 0.04$ \\
& $k_{\mathrm{bb}}\left(L_{39} / d_{10}^{2}\right)$ & $(3.6 \pm 0.2) \times 10^{-3}$ \\
Emission & $E_{\mathrm{gau}}(\mathrm{keV})$ & $6.45_{-0.29}^{+0.25}$ \\
& $\sigma(\mathrm{keV})$ & 0.85 \\
& $E W(\mathrm{eV})$ & $181 \pm 55$ \\
Fe XXVI & $E_{\mathrm{gau}}(\mathrm{keV})$ & $6.9 \pm 0.1$ \\
absorption & $\sigma(\mathrm{keV})$ & $<0.80$ \\
& $E W(\mathrm{eV})$ & $30 \pm 15$ \\
\hline
\end{tabular}

If the absorbing material is almost completely ionized, no strong absorption lines will be observed. Alternative explanations for the lack of deep absorption lines in 4U 1746-371 include an unusually low metallicity (e.g., Parmar et al. 1999), or a viewing angle outside of the ionized plasma.

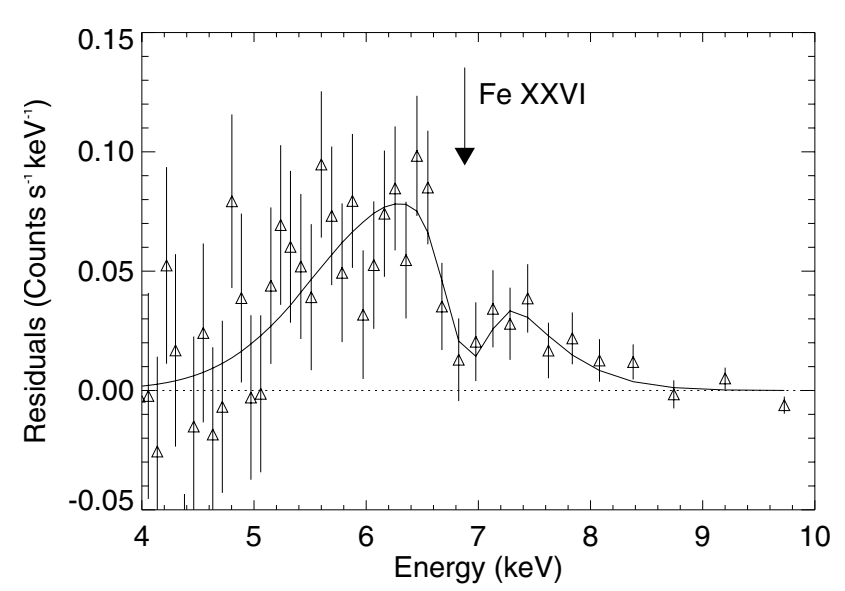

Fig. 5. 4U 1746-371: 4-10 keV persistent emission spectral residuals from the best-fit wabs (bb+pl+gau+gau) model (see Table 9) when the normalizations of the Gaussian emission feature and the Gaussian absorption feature are set to zero. The energy of the Fe XXVI absorption line at redshift 0 is indicated.

In order to investigate the effect of uncertainties in the ionizing continuum on the spectral parameters for the ionized absorbers, we performed fits to the XB 1254-690 spectra, using 
Table 10. MXB 1659-298: best-fits to the EPIC pn persistent and 5 dip spectra using the abs*xabs*(pl+bb)+abs*(gau+gau) model. The components of the continuum $(\mathrm{pl}+\mathrm{bb})$ are the same for all spectra and only the $N_{\mathrm{H}}$ of the neutral absorber, the line parameters, $N_{\mathrm{H}}^{\mathrm{xabs}}, \sigma_{v}$ and $\xi$ of the ionized absorber are individually fit for each spectrum. $F_{\mathrm{X}}$ is the $0.6-10 \mathrm{keV}$ absorbed flux. The $F W H M$ of the $6.4 \mathrm{keV}$ Gaussian emission line is constrained to be $\leq 2.0 \mathrm{keV}$. The $F W H M$ s of the $\sim 1 \mathrm{keV}$ Gaussian emission lines are constrained to be $\leq 0.2 \mathrm{keV}$.

\begin{tabular}{|c|c|c|c|c|c|c|}
\hline & Persistent & Dip 1 & Dip 2 & Dip 3 & Dip 4 & Dip 5 \\
\hline & & & & & & \\
\hline \multicolumn{7}{|l|}{ Parameter } \\
\hline & & & & & & \\
\hline$\Gamma$ & & & $1.96 \pm 0.03$ & & & \\
\hline$k_{\mathrm{pl}}\left(10^{44}\right.$ ph. $\left.\mathrm{s}^{-1} \mathrm{keV}^{-1}\right)$ & & & $51 \pm 2$ & & & \\
\hline & & & & & & \\
\hline$k T_{\mathrm{bb}}(\mathrm{keV})$ & & & $1.14 \pm 0.03$ & & & \\
\hline \multirow{2}{*}{$k_{\mathrm{bb}}\left(10^{11} \mathrm{~cm}^{2}\right)$} & & & $59 \pm 5$ & & & \\
\hline & & & & & & \\
\hline$N_{\mathrm{H}}\left(10^{22} \mathrm{~cm}^{-2}\right)$ & $0.306 \pm 0.003$ & $0.302 \pm 0.005$ & $0.34 \pm 0.01$ & $0.40 \pm 0.02$ & $0.47 \pm 0.04$ & $0.71 \pm 0.04$ \\
\hline$N_{\mathrm{H}}^{\mathrm{xabs}}\left(10^{22} \mathrm{~cm}^{-2}\right)$ & $11.1 \pm 0.6$ & $20 \pm 2$ & $19 \pm 4$ & $18 \pm 3$ & $24 \pm 3$ & $53 \pm 3$ \\
\hline $\log (\xi)\left(\mathrm{erg} \mathrm{cm} \mathrm{s}^{-1}\right)$ & $3.8 \pm 0.1$ & $3.7 \pm 0.2$ & $2.9 \pm 0.1$ & $2.55_{-0.06}^{+0.08}$ & $2.42_{-0.09}^{+0.06}$ & $2.42_{-0.06}^{+0.02}$ \\
\hline \multirow[t]{2}{*}{$\sigma_{\mathrm{v}}\left(\mathrm{km} \mathrm{s}^{-1}\right)$} & $700_{-350}^{+1000}$ & $150_{-100}^{+170}$ & $160_{-70}^{+100}$ & $140_{-40}^{+900}$ & $140_{-70}^{+0.00}$ & $110_{-30}^{+400}$ \\
\hline & & & & & & \\
\hline$E_{\text {gau }}(\mathrm{keV})$ & $6.4 \pm 0.2$ & & & & & \\
\hline$F W H M(\mathrm{keV})$ & 2 & & & & & \\
\hline \multirow[t]{2}{*}{$k_{\text {gau }}\left(10^{44} \mathrm{ph} \mathrm{s}^{-1}\right)$} & $0.53 \pm 0.08$ & & & & & \\
\hline & & & & & & \\
\hline$E_{\text {gau }}(\mathrm{keV})$ & $0.99 \pm 0.03$ & & & $0.96 \pm 0.03$ & $0.92 \pm 0.03$ & $0.93 \pm 0.02$ \\
\hline$F W H M(\mathrm{keV})$ & $0.11_{-0.07}^{+0.09}$ & & & $0.15 \pm 0.09$ & 0.2 & 0.2 \\
\hline$k_{\text {gau }}\left(10^{44} \mathrm{ph} \mathrm{s}^{-1}\right)$ & $0.4 \pm 0.2$ & & & $1.7_{-0.7}^{+1.0}$ & $3 \pm 2$ & $2.2 \pm 0.6$ \\
\hline \multirow{3}{*}{$\begin{array}{l}F_{\mathrm{X}}\left(10^{-10} \mathrm{erg} \mathrm{cm}^{-2} \mathrm{~s}^{-1}\right) \\
\chi_{v}^{2} \text { (d.o.f.) } \\
\text { Exposure }(\mathrm{ks})\end{array}$} & 9.7 & 8.9 & 7.9 & 6.6 & 5.4 & 2.9 \\
\hline & & & $1.22(1$ & & & \\
\hline & 11.8 & 2.16 & 0.48 & 0.56 & 0.35 & 1.33 \\
\hline
\end{tabular}

ionizing continua generated with, and without, the $5.9 \mathrm{keV}$ cutoff. Variations in $E_{\mathrm{c}}$ are likely to dominate the uncertainties in the ionizing continuum since the cutoff measurements cannot be obtained from fits to the pn spectra, due to the restricted energy range of this instrument $(0.6-10 \mathrm{keV})$, whilst the powerlaw indices are directly fit up to $10 \mathrm{keV}$. XB 1254-690 was chosen for this analysis since it has the lowest cutoff energy in Table 5, except for 4U 1746-371, which only exhibits shallow dips. The best-fit values of $N_{\mathrm{H}}^{\mathrm{xabs}}$ and $\sigma_{\mathrm{v}}$ are consistent within the errors for both ionization continua, while $\log (\xi)$ decreases by $\sim 0.3$ when the cutoff is not included. Since this represents an extreme range of the likely ionization continua, the actual uncertainties due to variations in the cutoff of the ionizing continuum are likely to be significantly smaller than this.

The changes in absorber properties for each source as it evolves from persistent to deep dipping do not strongly depend on the ionizing continuum used. Figure 8 shows the evolution of $\xi$ and $N_{\mathrm{H}}^{\mathrm{xabs}}$ from persistent to the deepest dip intervals for each source. A similar evolution of decreasing $\xi$ and increasing $N_{\mathrm{H}}^{\mathrm{xabs}}$ is observed for all sources, being the evolution stronger from persistent to Dip 2 stages than between dips. For EXO 0748-676, the evolution of the highly-ionized absorber is unusually small compared to the other sources. This could be explained if EXO 0748-676 were in a continuous dipping state (see above). Figure 9 shows the evolution of $N_{\mathrm{H}}$, with respect to the ionized absorber, $N_{\mathrm{H}}^{\mathrm{xabs}}$, from persistent to deepest dip intervals for each source. The increase of $N_{\mathrm{H}}$ is small in comparison to $N_{\mathrm{H}}^{\mathrm{xabs}}$. XB 1254-690 shows a particularly small increase in $N_{\mathrm{H}}$ compared to the other sources. This may indicate that we are viewing XB 1254-690 at an inclination angle such that the line of sight is not obscured by additional neutral material at any orbital phase and the dips result only from additional obscuration by the ionized absorber. Thus, the source may be being viewed relatively far from the plane of the accretion disk and only the ionized absorber significantly intercepts the line of sight. This special geometry and small changes in the size of the ionized absorber may explain the remarkable complete occasional disappearance of dipping activity from this source (Smale et al. 2002; Boirin \& Parmar 2003). If this picture is correct, the large changes in $N_{\mathrm{H}}$ observed from X 1624-490 during dips would indicate that we are viewing this source very close to the plane of the accretion disk.

Table 12 shows the changes in the properties of the neutral and highly-ionized absorbers from persistent (Dip 1 for X 1624-490) to deepest dip phases. We include in this table results for XB 1323-619 derived from the spectral fits presented in Boirin et al. (2005). These authors fixed the normalizations of the dipping spectra to that of the persistent spectrum, thus a larger change in the neutral absorber column may be necessary to account for the changes when compared to fitting 

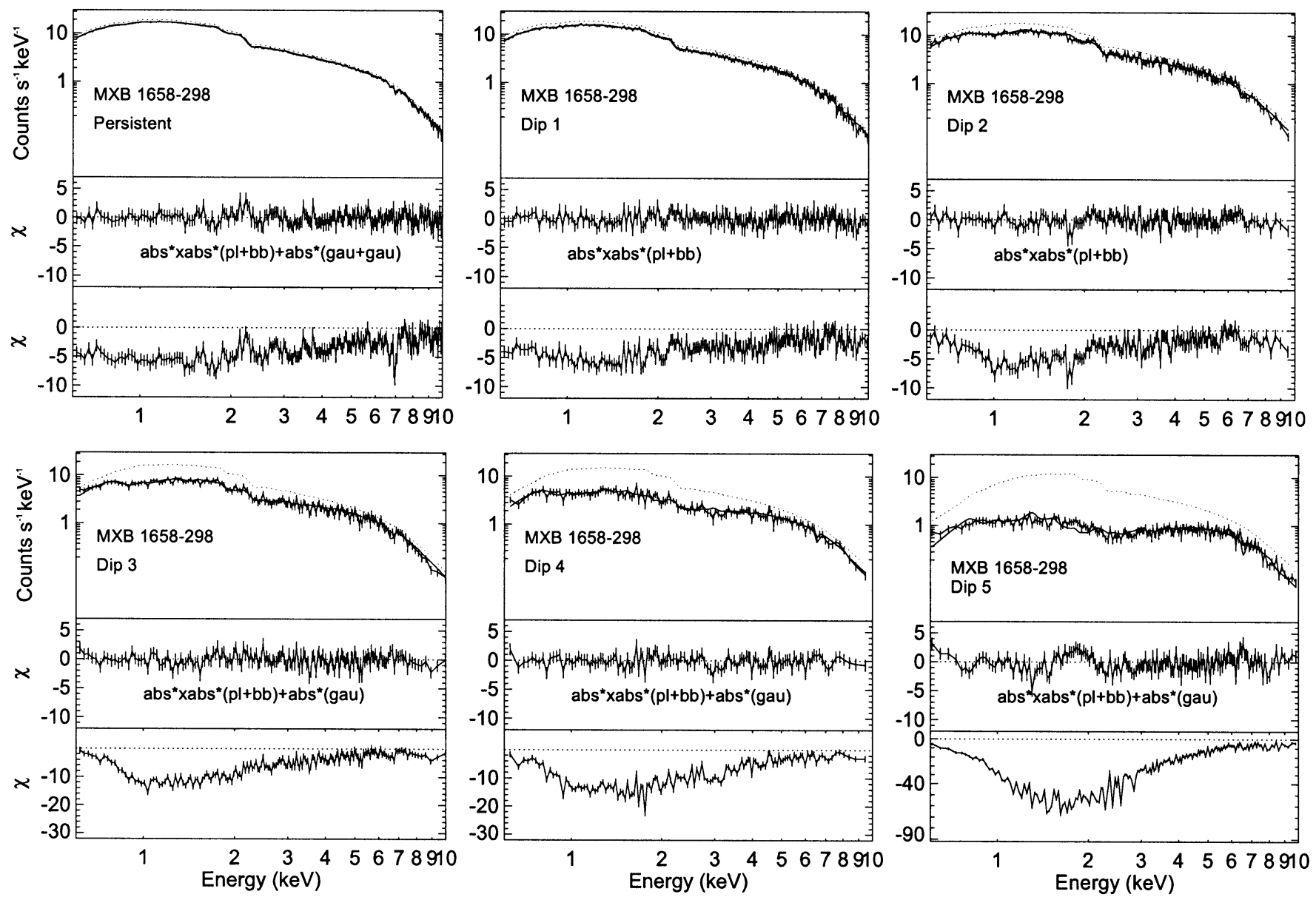

Fig. 6. MXB 1659-298: EPIC pn persistent and dipping spectra fit with a power-law (pl) and blackbody model (bb), modified by absorption from neutral (abs) and ionized (xabs) material and 2 narrow emission lines (gau), modified by absorption from neutral material (abs) (see Table 10). The dotted lines show the models when the column of the ionized absorber is set to 0 . The middle panels show the residuals in units of standard deviations from the above model. The lower panels show residuals when $N_{\mathrm{H}}^{\mathrm{xabs}}$ is set to 0 .

simultaneously the persistent and dipping spectra, as was done for the other sources. It is interesting that the eclipsing binaries EXO 0748-676 and MXB 1659-298 (together with the noneclipsing system X 1624-490) show the largest change in $N_{\mathrm{H}}$. This suggests that the size of the change in $N_{\mathrm{H}}$ may be related to the inclination angle. This supports the idea that we are seeing X 1624-490 and XB 1254-690 very close to, and relatively far from, the planes of the accretion disks. Alternative explanations for the large increase in $N_{\mathrm{H}}$ for X 1624-490 include the high luminosity of this source, uncertainties in modeling the dust scattering halo, or an absorber rich in blobs of neutral material. Furthermore, XB 1254-690 and XB 1323-619, which show the smallest relative changes of $N_{\mathrm{H}}$, have also the less deep dips, consistent with viewing the sources relatively far from the disk plane. We do not find a correlation between the dip depth and the change in $N_{\mathrm{H}}^{\mathrm{xabs}}$ or $\log (\xi)$. EXO 0748-676 and MXB 1659-298, which show the deepest dips, have also the lowest values of $\xi$ during the deepest dips. The change in $N_{\mathrm{H}}^{\mathrm{xabs}}$ is approximately the same for all the sources except EXO 0748-676. This is shown in Fig. 10 by plotting the changes of the neutral and ionized absorbers versus $P_{\text {orb }}$ and $L$ of the studied sources. Note that we have excluded X 1624-490 from this figure, whose persistent emission has not been analyzed, and EXO 0748-676, which may be continuously dipping. In contrast to $N_{\mathrm{H}}^{\mathrm{xabs}}$, the change of $\log (\xi)$ appears to increase with the source $P_{\text {orb }}$ and $L$.

It is interesting to compare the values of the ionization parameter for the different sources. Since $\xi=L / n_{\mathrm{e}} r^{2}$, the relation between $\xi$ and $L$ can provide information about the distance between the absorbing material and the ionizing source, $r$. Figure 11 shows the ionized absorber parameters $\log (\xi)$ and $N_{\mathrm{H}}^{\mathrm{xabs}}$ as a function of $P_{\mathrm{orb}}$ and source luminosity $L$ for the persistent emission. Note that we excluded X 1624-490 and EXO 0748-676 from this figure (see above). The $\log (\xi)$ and $N_{\mathrm{H}}^{\mathrm{xabs}}$ of the absorbers in our sample appear to increase with luminosity. Since the luminosity is proportional to the mass accretion rate, this suggests that the column of the ionized absorber increases with the mass accretion rate. MXB 1659-298 has a lower $\xi$ than the less luminous XB 1254-690. This indicates that either $n_{\mathrm{e}}$ or $r$ are much larger for MXB 1659-298 than for XB 1254-690. Assuming a constant $n_{\mathrm{e}}$, the evolution of $\log (\xi)$ with $\log L$ suggests that for low $L$ sources, the change of $r$ is small compared to the $L$, while for sources with $L \gtrsim 10^{37} \mathrm{erg} \mathrm{s}^{-1}, r$ increases faster than $L$. There may also be 
Table 11. X 1624-490: best-fits to the EPIC pn 5 dip spectra using the $a b s_{1} * x_{a b s} * e^{-\tau}(b b+p l)+a b s_{2} *\left(1-e^{-\tau}\right) *(b b+p l)$ model. The components of the continuum $(\mathrm{pl}+\mathrm{bb})$, the column density of the halo neutral absorber and $\tau$ are the same for all spectra and only the $N_{\mathrm{H}}$ of the neutral absorber, and $N_{\mathrm{H}}^{\mathrm{xabs}}, \sigma_{v}$ and $\xi$ of the ionized absorber are individually fit for each spectrum. $F_{\mathrm{X}}$ is the $0.6-10 \mathrm{keV}$ absorbed flux. $\Gamma$ is constrained to be $\geq 1.90$.

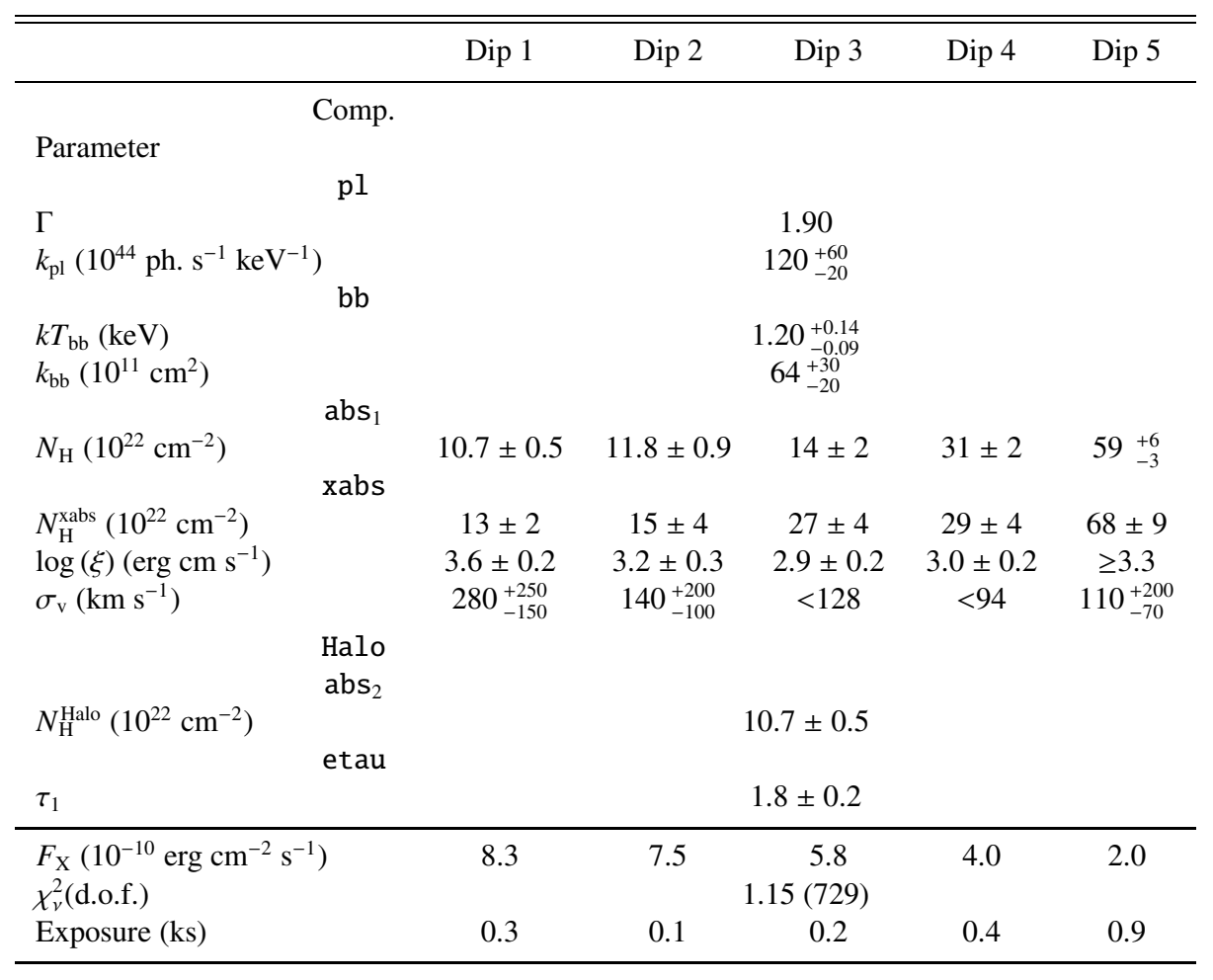

a correlation between $\log (\xi)$ or $N_{\mathrm{H}}^{\mathrm{xabs}}$ and $P_{\mathrm{orb}}$. The increase of $\log (\xi)$ with $\log \left(P_{\text {orb }}\right)$ indicates that the system period is less important than the luminosity of the system for the degree of ionization of the plasma, since otherwise systems with small $P_{\text {orb }}$, and correspondingly smaller distances between the source and the absorber, should ionize more effectively the absorbing material.

We can compare the thickness of the slab of ionized absorbing material, $d$, with the distance between the ionizing source and the slab, $r$. Since $\xi=L / n_{\mathrm{e}} r^{2}$ and $n_{\mathrm{e}} \sim N_{\mathrm{H}}^{\mathrm{xabs}} / d$, we can calculate the ratio, $d / r$, as $\xi N_{\mathrm{H}}^{\mathrm{xabs}} r / L$. We estimated a range of possible ratios for persistent and deepest dip emission taking into account the uncertainties in the determination of $r$ and $L$. We considered the circularization radius as the minimum possible value for $r$ and $0.8 r_{L}$, where $r_{L}$ is the radius of the Roche lobe, as the maximum possible value for $r$. We assumed that the accretion occurs via Roche lobe overflow and calculated the radii from $P_{\text {orb }}$ (Eqs. (4.16) from Frank et al. 1992, and (4.18) from Shore et al. 1994). In the determination of $L$ the largest uncertainty is given by the uncertainty in the distance. We calculated an average value for the distance from all the values given in the literature and considered an uncertainty of $\pm 3 \mathrm{kpc}$ for each source (see Table 13).

We note that during dipping emission EXO 0748-676 and MXB 1659-298, which have relatively cool absorbers $(\log (\xi)$ $\lesssim 2.5$ ) and show deep and sharp dips, have $d<r$, while the sources with more ionized absorbers have $d \sim r$. During persistent emission, all the values of $d$ are consistent with $r$ (taking uncertainties into account) except for XB 1916-053 and EXO 0748-676, which show small values of $d / r(\sim 0.04-$ $0.6)$ and the smallest values of $\xi$. This may indicate that the material responsible for the ionized absorption seen during persistent emission is clumpy and located at the outer edge of the accretion disk for XB 1916-053 and EXO 0748-676 and distributed on a significant fraction of the accretion disk for the other sources.

\section{Discussion}

We have demonstrated that the complex changes in the 0.6-10 keV continuum and absorption lines during dips from most of the LMXBs studied may be self-consistently understood as resulting from large increases in $N_{\mathrm{H}}^{\mathrm{xabs}}$ and decreases in $\xi$ of a highly-ionized absorber, together with much smaller increases in the $N_{\mathrm{H}}$ of a neutral absorber. These changes are similar to those found by Boirin et al. (2005) for XB 1323-619. We do not need to invoke unusual abundances or partial coverage of an extended emission region to account for these changes.

Narrow X-ray absorption lines were first detected from the superluminal jet sources GRO J1655-40 (Ueda et al. 1998; Yamaoka et al. 2001) and GRS 1915+105 (Kotani et al. 2000; Lee et al. 2002). GROJ1655-40 has been observed to undergo deep absorption dips (Kuulkers et al. 1998) consistent with observing the source at an inclination angle of $60^{\circ}-$ $75^{\circ}$ (e.g., Orosz \& Bailyn 1997; Van der Hooft et al. 1998). Recently Chandra HETGS observations of the black hole 

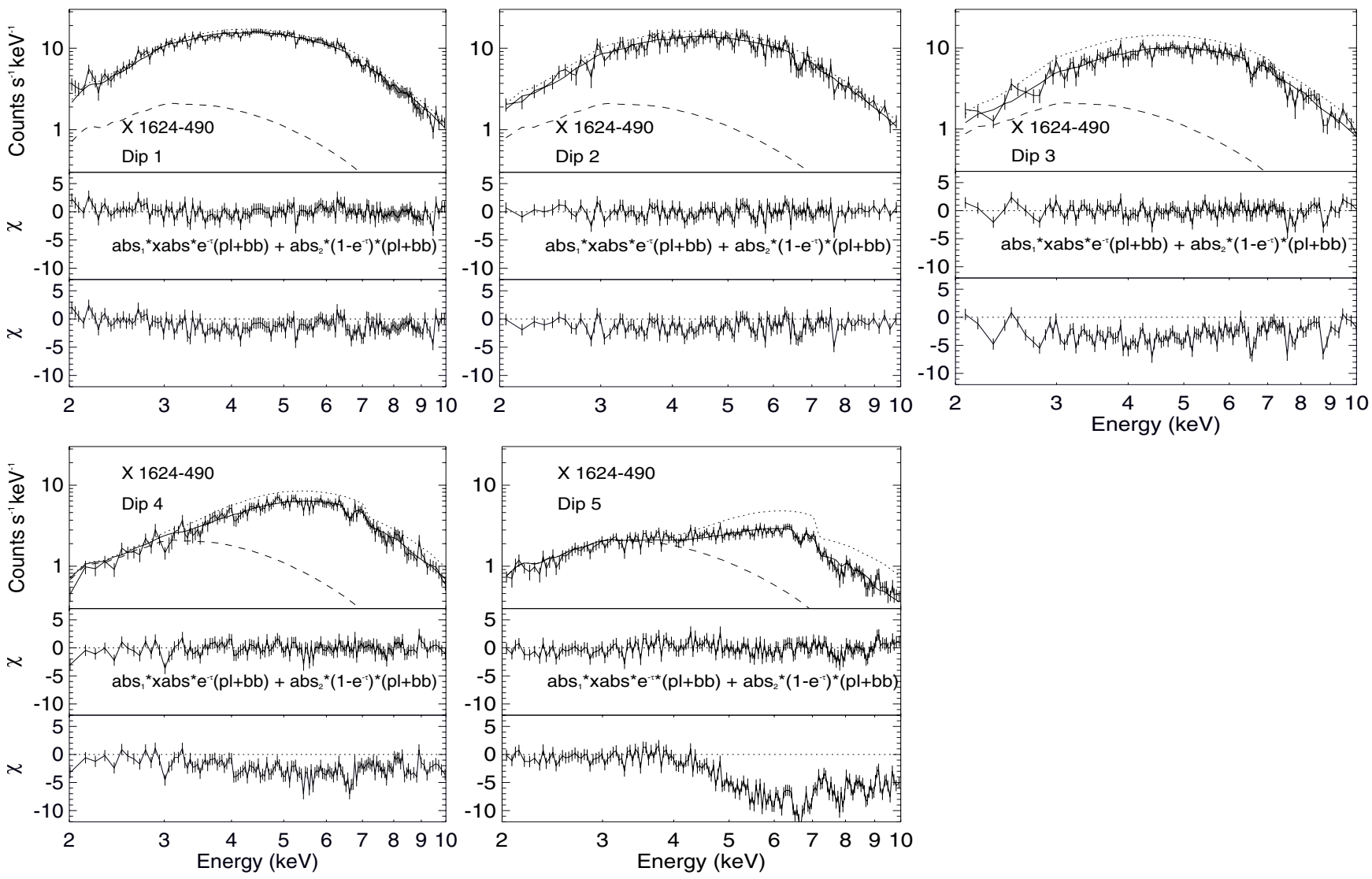

Fig. 7. X 1624-490: EPIC pn dipping spectra fit with the model of Table 11. The dotted lines show the models when the column of the ionized absorber is set to 0 . The dashed lines show the constant halo component. The middle panels show residuals in units of standard deviations from the above model. The lower panels show residuals when the $N_{\mathrm{H}}^{\mathrm{xabs}}$ of the ionized absorber is set to 0 .

Table 12. The persistent (Dip 1 for X 1624-490) values of $N_{\mathrm{H}}$ (Col. 2), $N_{\mathrm{H}}^{\mathrm{xabs}}(\mathrm{Col} .6)$ and $\log (\xi)(\mathrm{Col} .8)$ and the changes in $N_{\mathrm{H}}(\mathrm{Col} .3)$ and $N_{\mathrm{H}}^{\mathrm{xabs}}(\mathrm{Col} .7)$ from persistent to the deepest dip intervals observed for each source. $N_{\mathrm{Hgal}}$ is the averaged interstellar value for the $0.5 \mathrm{region}$ in the sky containing the source (Dickey \& Lockman 1990). $\Delta N_{\mathrm{H}} /\left(N_{\mathrm{Hpers}}-N_{\mathrm{Hgal}}\right)$ is the relative change in $N_{\mathrm{H}}$ local to the source from persistent to the deepest dip interval. Col. 9 shows the value of $\log (\xi)$ during the deepest dip for each source. $N_{\mathrm{H}}$ for EXO $0748-676$ is constrained to be $\geq 1.1 \times 10^{21}$ atom $\mathrm{cm}^{-2}$. All values of $N_{\mathrm{H}}, N_{\mathrm{H}}^{\mathrm{xabs}}$ and their changes are expressed in units of $10^{22}$ atom $\mathrm{cm}^{-2}$.

\begin{tabular}{lccccccccc}
\hline \hline LMXB & $N_{\text {Hpers }}$ & $\Delta N_{\mathrm{H}}$ & $N_{\mathrm{Hgal}}$ & $\begin{array}{c}\Delta N_{\mathrm{H}} / \\
\left(N_{\mathrm{Hpers}}-N_{\mathrm{Hgal}}\right)\end{array}$ & $N_{\mathrm{H}}^{\mathrm{xabs}}$ pers & $\Delta N_{\mathrm{H}}^{\text {xabs }}$ & $\log (\xi)_{\text {pers }}$ & $\log (\xi)_{\text {dip }}$ & $\begin{array}{c}\text { Dip } \\
\text { depth }\end{array}$ \\
\hline XB 1916-053 & $0.432 \pm 0.002$ & $0.46 \pm 0.07$ & 0.27 & $2.8 \pm 0.4$ & $4.2 \pm 0.5$ & $50 \pm 3$ & $3.05 \pm 0.04$ & $2.522_{-0.06}^{+0.02}$ & $80 \%$ \\
XB 1323-619 & $3.50 \pm 0.02$ & $0.7 \pm 0.2$ & 1.57 & $0.4 \pm 0.1$ & $3.8 \pm 0.4$ & $33 \pm 2$ & $3.9 \pm 0.1$ & $3.13 \pm 0.07$ & $75 \%$ \\
EXO 0748-676 & 0.11 & $0.13_{-0.05}^{+0.09}$ & 0.11 & $\infty$ & $3.5 \pm 0.2$ & $12.0 \pm 0.5$ & $2.45 \pm 0.02$ & $2.26 \pm 0.03>85 \%$ \\
XB 1254-690 & $0.346 \pm 0.002$ & $0.04 \pm 0.01$ & 0.31 & $1.0 \pm 0.3$ & $8.4 \pm 0.3$ & $39 \pm 3$ & $4.3 \pm 0.1$ & $2.94 \pm 0.05$ & $50 \%$ \\
MXB 1659-298 & $0.306 \pm 0.003$ & $0.40 \pm 0.04$ & 0.19 & $3.5 \pm 0.4$ & $11.1 \pm 0.6$ & $42 \pm 3$ & $3.8 \pm 0.1$ & 2.42 \\
X 1624-490 & $10.7 \pm 0.5$ & $48_{-3}^{+6}$ & 2.02 & $5.77_{-0.4}^{+0.7}$ & $13 \pm 2$ & $55 \pm 9$ & $3.6 \pm 0.2$ & $\geq 3.3$ & $80 \%$ \\
\hline
\end{tabular}

\footnotetext{
${ }^{a}$ Values for XB 1323-619 are derived from the spectral fits in Boirin et al. (2005).

${ }^{b}$ The changes for X 1624-490 are calculated between the Dip 1 and Dip 5 stages.
}

candidates GX339-4, XTEJ1650-500 and H 1743-322 (Miller et al. 2004a,b) have revealed the presence of variable, blue-shifted, highly-ionized absorption features which are interpreted as evidence for outflows. While Fe XXV and Fe XXVI features are present in the H 1743-322 spectrum, GX 339-4 and XTE J1650-500 show O VIII and Ne IX or Ne II features from less ionized material. These features suggest that a warm absorber analogous to those seen in many Seyfert galaxies is present in systems such as GX 339-4 and XTEJ1650-500 (Miller et al. 2004a). In contrast, Miller et al. (2004b) propose for H 1743-322 a highly-ionized absorber, which may be a precursor to the cooler outflows observed in GX339-4 and XTE J1650-500. XMM-Newton observations of the LMXB GX 13+1 revealed absorption features due to CaXX, Fe XXV and Fe XXVI (Sidoli et al. 2002) and an ionized outflow with a velocity of $\sim 400 \mathrm{~km} \mathrm{~s}^{-1}$ was reported by Ueda et al. (2004). 


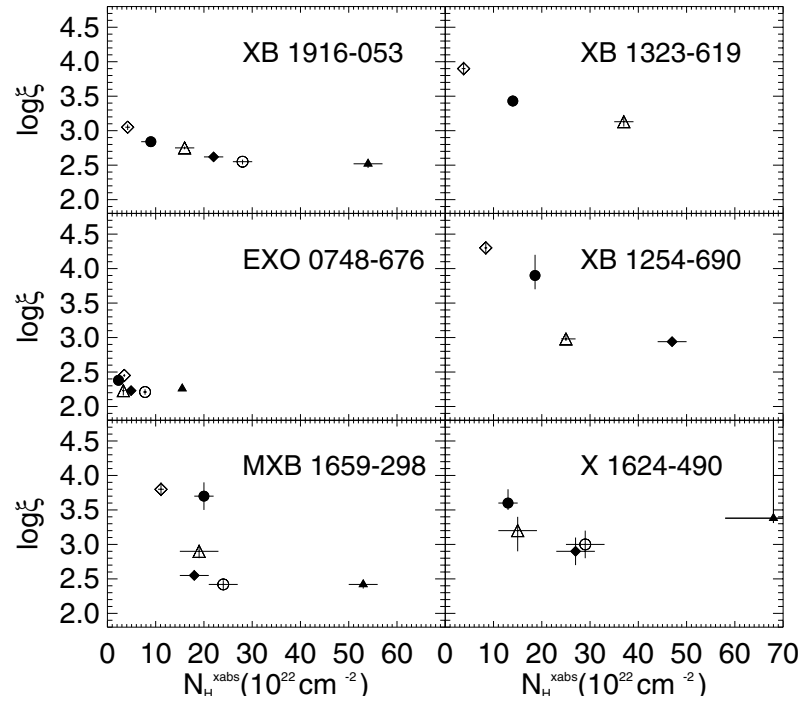

Fig. 8. Evolution of $\log (\xi)$ and $N_{\mathrm{H}}^{\mathrm{xabs}}$ for the LMXBs studied in this paper. Empty diamonds, filled circles, empty triangles, filled diamonds, empty circles and filled triangles indicate the persistent and Dip 1 to Dip 5 intervals, respectively.

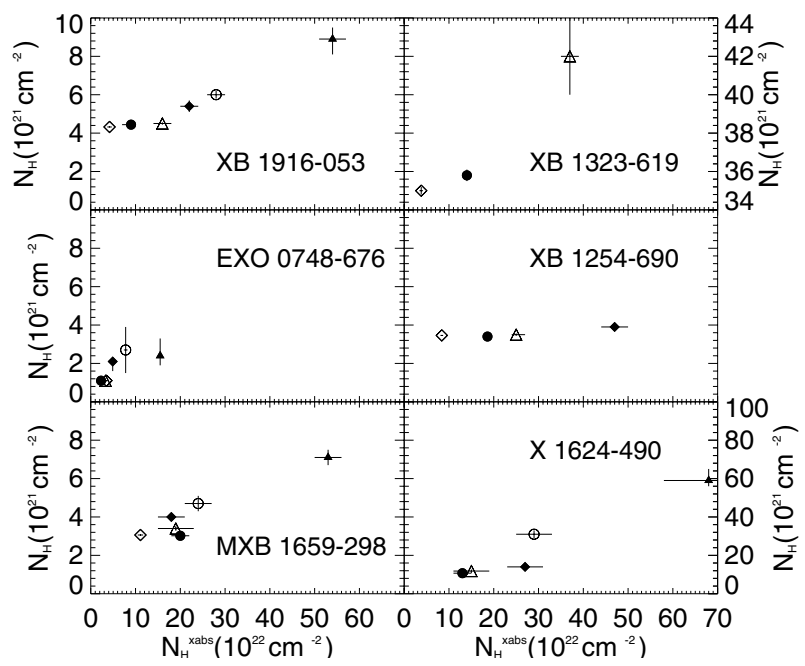

Fig. 9. Evolution of the column densities of the neutral and ionized absorbers, $N_{\mathrm{H}}$, and $N_{\mathrm{H}}^{\mathrm{xabs}}$, for the LMXBs studied in this paper. Empty diamonds, filled circles, empty triangles, filled diamonds, empty circles and filled triangles indicate the persistent and Dip 1 to Dip 5 intervals, respectively.

Blueshifted outflows have not been detected from any of the dipping LMXBs (Sidoli et al. 2001; Parmar et al. 2002; Boirin \& Parmar 2003; Boirin et al. 2004, 2005). However, these results are all obtained with the EPIC which has a factor $\sim 4$ poorer energy resolution than the HETGS at $6 \mathrm{keV}$ limiting the sensitivity to shifts $\gtrsim 1000 \mathrm{~km} \mathrm{~s}^{-1}$. Since dipping sources are simply normal LMXBs viewed from close to the orbital plane, this also implies that ionized absorbers, frequent in microquasars and AGNs (e.g., Reynolds 1997; Blustin et al. 2005), are also a common feature of LMXBs. Outside of the dips, the properties of the absorption lines do not appear to vary strongly with orbital phase. This suggests that the ionized plasma in

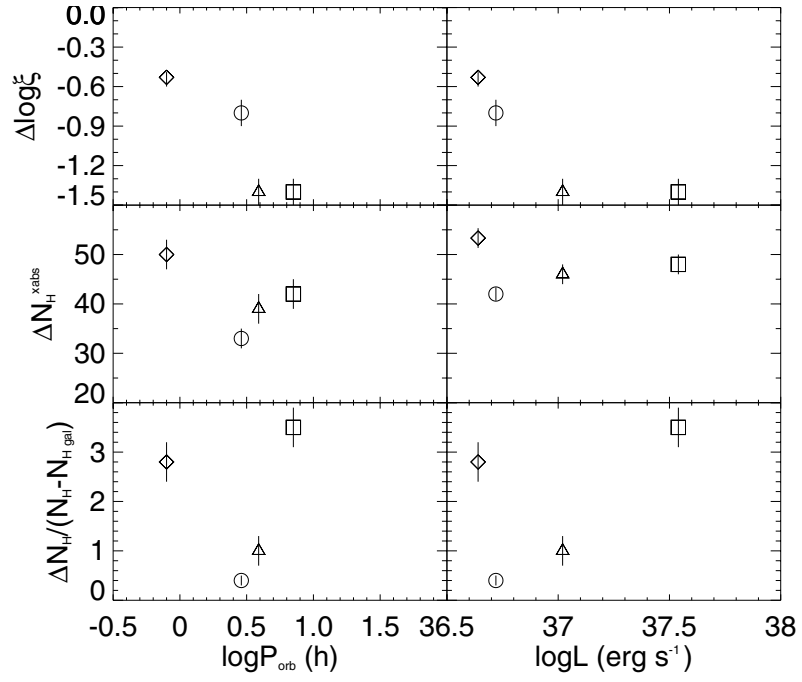

Fig. 10. Changes of $\log (\xi), N_{\mathrm{H}}^{\mathrm{xabs}}$ and $N_{\mathrm{H}}$ from persistent to deepest dip emission versus orbital period and luminosity for the LMXBs studied here, except EXO 0748-676 and X 1624-490 (see text). The change of $N_{\mathrm{H}}$ is expressed relative to the local persistent value of $N_{\mathrm{H}}$. Diamonds, circles, triangles and squares represent XB 1916-053, XB 1323-619, XB 1254-690 and MXB 1659-298, respectively.

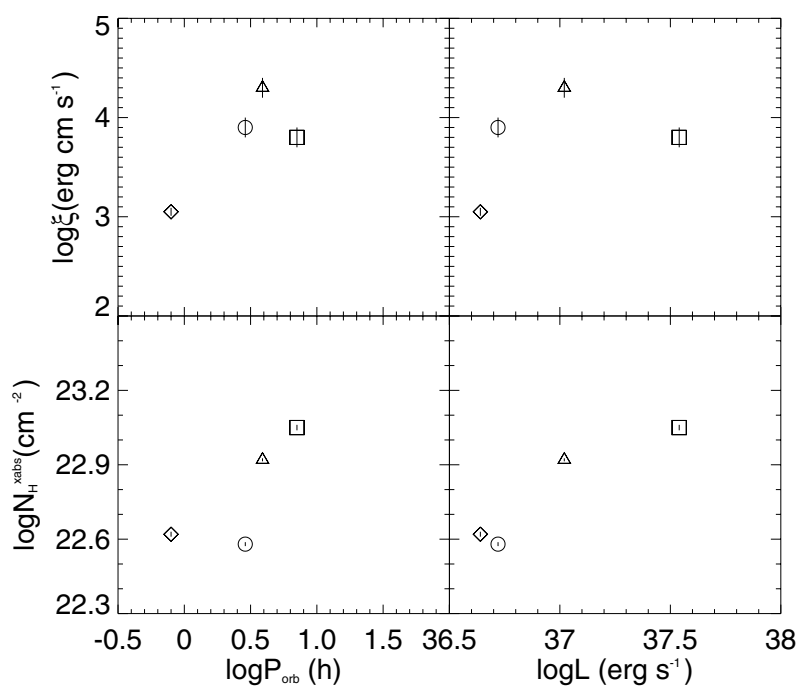

Fig. 11. Values of $\log (\xi)$ and $\log N_{\mathrm{H}}^{\mathrm{xabs}}$ for the ionized absorbers during persistent emission versus $P_{\text {orb }}$, and $L$, for the LMXBs studied here, except EXO 0748-676 and X 1624-490 (see text). Diamonds, circles, triangles and squares represent XB 1916-053, XB 1323-619, XB 1254-690 and MXB 1659-298, respectively.

LMXBs has a cylindrical geometry with a maximum column density close to the plane of the accretion disk.

The spectral changes during dips from LMXBs are often modeled using the "progressive covering", or "complex continuum" approach (e.g., Church \& Balucinska-Church 1995; Barnard et al. 2001). There the X-ray emission is assumed to originate from a point-like blackbody, or disk-blackbody component, together with an extended power-law component. This approach models the spectral changes during dipping intervals by the partial and progressive covering of the extended component by an opaque absorber. We have self-consistently 
Table 13. Slab thickness of ionized absorbing material, $d$, with respect to the size of the accretion disk, $r$, during persistent and deepest dip emission for all the studied sources. Col. 2 shows estimates of $r$ obtained from $P_{\text {orb }}$ and Col. 3 estimates of the source distances (see text).

\begin{tabular}{|c|c|c|c|c|}
\hline LMXB & $\begin{array}{c}\mathrm{r} \\
\left(10^{10} \mathrm{~cm}\right)\end{array}$ & $\begin{array}{c}\text { dist } \\
(\mathrm{kpc})\end{array}$ & $(\mathrm{d} / \mathrm{r})_{\text {pers }}$ & $(\mathrm{d} / \mathrm{r})_{\text {dip }}$ \\
\hline XB 1916-053 & $1.1-2.0$ & $6-12$ & $0.07-0.5$ & $0.3-2$ \\
\hline XB 1323-619 & $1.5-4.2$ & $7-13$ & $0.5-5$ & $0.9-8$ \\
\hline EXO 0748-676 & $1.7-4.9$ & $5-11$ & $0.04-0.6$ & $0.1-2$ \\
\hline XB 1254-690 & $1.7-4.9$ & $8-14$ & $1-12$ & $0.3-3$ \\
\hline MXB 1659-298 & $2.1-7.0$ & $10-16$ & $0.4-3$ & $0.07-0.6$ \\
\hline X $1624-490$ & $3.8-13.3$ & $12-18$ & $0.3-2$ & $0.8-6$ \\
\hline
\end{tabular}

demonstrated that changes in the properties of an ionized absorber provide an alternative explanation for the overall spectral changes during dips from all the dipping LMXBs studied by XMM-Newton. Further investigations and particularly the high spectral resolution observations of LMXBs expected from Astro-E2 should allow many of the features predicted by the ionized absorber model to be characterized and outflowing plasmas in LMXBs to be studied.

Acknowledgements. Based on observations obtained with XMMNewton, an ESA science mission with instruments and contributions directly funded by ESA member states and the USA (NASA). M. Díaz Trigo acknowledges an ESA Fellowship. SRON is supported financially by NWO, the Netherlands Organization for Scientific Research. We thank the anonymous referee for helpful comments.

\section{References}

Angelini, L., \& White, N. E. 2003, ApJ, 586, L71

Angelini, L., Parmar, A., \& White, N. 1997, in ASP Conf. Ser., 685

Arnaud, K. A. 1996, in Astronomical Data Analysis Software and Systems V, ASP Conf. Ser., 101, 17

Bałucińska-Church, M., Church, M. J., Oosterbroek, T., et al. 1999, A\&A, 349, 495

Bałucińska-Church, M., Humphrey, P. J., Church, M. J., \& Parmar, A. N. 2000, A\&A, 360, 583

Bałucińska-Church, M., Church, M. J., \& Smale, A. P. 2004, MNRAS, 347, 334

Barnard, R., Balucińska-Church, M., Smale, A. P., \& Church, M. J. 2001, A\&A, 380, 494

Blustin, A. J., Page, M. J., Fuerst, S. V., Branduardi-Raymont, G., \& Ashton, C. E. 2005, A\&A, 431, 111

Boirin, L., \& Parmar, A. N. 2003, A\&A, 407, 1079

Boirin, L., Méndez, M., Díaz Trigo, M., Parmar, A. N., \& Kaastra, J. S. 2005, A\&A, 436, 195

Boirin, L., Parmar, A. N., Barret, D., Paltani, S., \& Grindlay, J. E. 2004, A\&A, 418, 1061

Church, M. J. \& Balucinska-Church, M. 1995, A\&A, 300, 441
Church, M. J., Parmar, A. N., Balucinska-Church, M., et al. 1998, A\&A, 338, 556

Cottam, J., Paerels, F., \& Mendez, M. 2002, Nature, 420, 51

Courvoisier, T. J. L., Parmar, A. N., Peacock, A., \& Pakull, M. 1986, ApJ, 309, 265

Den Herder, J. W., Brinkman, A. C., Kahn, S. M., et al. 2001, A\&A, 365, L7

Dickey, J. M., \& Lockman, F. 1990, ARA\&A

Ferland, G. J. 2003, ARA\&A, 41, 517

Frank, J., King, A. R., \& Raine, D. J. 1992, Accretion power in astrophysics (Cambridge and New York, Cambridge University Press)

Jansen, F., Lumb, D., Altieri, B., et al. 2001, A\&A, 365, L1

Kaastra, J. S., Mewe, R., \& Nieuwenhuijzen, H. 1996, in UV and $\mathrm{X}$-ray Spectroscopy of Astrophysical and Laboratory Plasmas, Frontiers science series, 15, 411

Kallman, T., \& Bautista, M. 2001, ApJS, 133, 221

Kotani, T., Ebisawa, K., Dotani, T., et al. 2000, ApJ, 539, 413

Kuulkers, E., Wijnands, R., Belloni, T., et al. 1998, ApJ, 494, 753

Lee, J. C., Reynolds, C. S., Remillard, R., et al. 2002, ApJ, 567, 1102

Miller, J. M., Raymond, J., Fabian, A. C., et al. 2004a, ApJ, 601, 450

Miller, J. M., Raymond, J., Homan, J., et al. 2004b, [arXiv: astro-ph/0406272]

Morrison, R., \& McCammon, D. 1983, ApJ, 270, 119

Oosterbroek, T., Parmar, A. N., Sidoli, L., in't Zand, J. J. M., \& Heise, J. 2001, A\&A, 376, 532

Orosz, J. A., \& Bailyn, C. D. 1997, ApJ, 477, 876

Parmar, A. N., White, N. E., Giommi, P., \& Gottwald, M. 1986, ApJ, 308, 199

Parmar, A. N., Oosterbroek, T., Guainazzi, M., et al. 1999, A\&A, 351, 225

Parmar, A. N., Oosterbroek, T., Boirin, L., \& Lumb, D. 2002, A\&A, 386, 910

Reynolds, C. S. 1997, MNRAS, 286, 513

Ritter, H., \& Kolb, U. 2003, A\&A, 404, 301

Shore, S. N., Livio, M., \& van den Heuvel, E. P. J. 1994, Interacting Binaries (Springer Verlag)

Sidoli, L., Oosterbroek, T., Parmar, A. N., Lumb, D., \& Erd, C. 2001, A\&A, 379, 540

Sidoli, L., Parmar, A. N., Oosterbroek, T., \& Lumb, D. 2002, A\&A, 385,940

Sidoli, L., Parmar, A. N., \& Oosterbroek, T. 2005, A\&A, 429, 291

Smale, A. P., Mukai, K., Williams, O. R., Jones, M. H., \& Corbet, R. H. D. 1992, ApJ, 400, 330

Smale, A. P., Church, M. J., \& Bałucińska-Church, M. 2002, ApJ, 581, 1286

Strüder, L., Briel, U., Dennerl, K., et al. 2001, A\&A, 365, L18

Turner, M. J. L., Abbey, A., Arnaud, M., et al. 2001, A\&A, 365, L27

Ueda, Y., Inoue, H., Tanaka, Y., et al. 1998, ApJ, 492, 782

Ueda, Y., Murakami, H., Yamaoka, K., Dotani, T., \& Ebisawa, K. 2004, ApJ, 609, 325

Van der Hooft, F., Heemskerk, M. H. M., Alberts, F., \& van Paradijs, J. 1998, A\&A, 329, 538

Verner, D. A., \& Yakovlev, D. G. 1995, A\&AS, 109, 125

Verner, D. A., Verner, E. M., \& Ferland, G. J. 1996, Atomic Data and Nuclear Data Tables, 64, 1

White, N. E., \& Swank, J. H. 1982, ApJ, 253, L61

Yamaoka, K., Ueda, Y., Inoue, H., et al. 2001, PASJ, 53, 179 